\title{
Dissipativity preserving model reduction by retention of trajectories of minimal dissipation
}

\author{
Harry L. Trentelman • Ha Binh Minh • \\ Paolo Rapisarda
}

Received: 9 October 2007 / Accepted: 18 August 2009 / Published online: 16 September 2009

(C) The Author(s) 2009. This article is published with open access at Springerlink.com

\begin{abstract}
We present a method for model reduction based on ideas from the behavioral theory of dissipative systems, in which the reduced order model is required to reproduce a subset of the set of trajectories of minimal dissipation of the original system. The passivity-preserving model reduction method of Antoulas (Syst Control Lett 54:361-374, 2005) and Sorensen (Syst Control Lett 54:347-360, 2005) is shown to be a particular case of this more general class of model reduction procedures.
\end{abstract}

Keywords Model reduction - Strictly dissipative systems - Behaviors - Minimal dissipation - Driving variable representation - Output nulling representations . Nevanlinna interpolation problem

\section{Introduction}

Model reduction aims at finding a system that approximates a given one and has lower complexity than the original, with the complexity being measured by its McMillan degree, i.e. the minimal dimension of the state space of the model. In the linear setting, classical model reduction methods are balancing (see [13]), Padé approximation (see [4]), moment-matching (see [24,10]), and $H_{\infty}$-approximation (see [8]). An up-to-date

H. L. Trentelman (凶)

Mathematics Institute, University of Groningen, P.O. Box 800, 9700 AV Groningen, The Netherlands e-mail: h.1.trentelman@math.rug.nl

H. B. Minh

Department of Mathematics, Hanoi University of Technology, 1 Dai Co Viet Str., Hanoi, Vietnam e-mail: habinhminh@yahoo.com

P. Rapisarda

ISIS Group, School of Electronics and Computer Science, University of Southampton,

SO17 1BJ Southampton, UK

e-mail: pr3@ecs.soton.ac.uk 
and exhaustive source on the problem of model reduction and approximation is given in [1].

Usually, besides the reduction in complexity of the original model, preservation of certain properties of the original model is required. An example of this is preservation of stability. However, often it is also demanded that the reduced model retains other characteristics of the original system, passivity being one of them. Several methods for model reduction with stability and passivity preservation have been introduced in the past, see for example [5-7,14,15,25].

Recently, Antoulas [2] and Sorensen [19] have presented a new technique and efficient numerical algorithms to perform model reduction with passivity and stability preservation. The novel approach pioneered by Antoulas in [2] is based on the idea of combining Krylov projection methods with positive-real interpolation techniques; the reduced order model is obtained by interpolating at a subset of the spectral zeros of the original system. In the closely related paper [19], Sorensen shows that for all practical purposes there is no need for explicit interpolation in the implementation: rather, the reduced order model can be found by computing a suitable basis for the stable invariant subspace of a Hamiltonian matrix associated with the system. This idea renders Antoulas' model reduction method applicable also to systems with large McMillan degree.

The purpose of the present paper is to present a different point of view on the method of [19] using ideas from the behavioral theory of dissipative systems. We show that the model reduction approach of Sorensen can be interpreted as special case of a general method for model reduction applicable to dissipative systems. For a given dissipative behavior we introduce the subbehavior of trajectories that are in a sense local minima of dissipation. Next, for the reduced order approximation we require that a particular part of its subbehavior of minimal dissipation is contained in the subbehavior of minimal dissipation of the original system: the approximating behavior 'inherits' this part of the subbehavior of minimal dissipation from the original system. We will call this technique model reduction by retention of trajectories of minimal dissipation.

In our setting, the original system will be given as the behavior of a linear, timeinvariant differential system. We assume that the behavior is dissipative with respect to a given supply rate. The complexity of the behavior is measured by its McMillan degree. The problem that we will study in this paper is to find, for a given positive integer $\mathrm{k}$ less than the McMillan degree of the original behavior, a (approximating) behavior: (1) whose McMillan degree is less than or equal to k, (2) that has the same number of inputs as the original behavior, (3) that is again dissipative with respect to the given supply rate, and (4) that retains (or inherits) a maximal number of a priori given antistable trajectories of minimal dissipation of the original behavior. Interpreted in this sense, the method of passivity preserving model reduction as initiated by Antoulas and Sorensen has the same heuristic flavor as the method of positive real balancing (see [5]), where it can be argued that the reduced order model is obtained by deleting typically that part of the system along which a relatively large amount of dissipation takes place.

We will establish an algorithmic procedure to compute, for a given behavior represented in driving variable representation, a reduced order behavior that solves the 
problem stated above. Subsequently, we will show that a certain transfer matrix associated with our reduced order behavior is in fact a solution of a Nevanlinna-type tangential interpolation problem (see also [11]). In fact, the transfer matrix of the reduced order behavior will turn out to interpolate the transfer matrix of the original behavior in certain directions, with interpolation points at some of the spectral zeros and their mirror images in the imaginary axis of the original behavior.

The outline of this paper is as follows. In Sect. 2, we review the basic material on behaviors that we need in this paper. Section 3 reviews the concepts of dissipativity, storage function, and dissipation function. Also, in this section the notion of subbehavior of minimal dissipation is introduced and elaborated. In Sect. 4, we state the exact problem that this paper deals with: the problem of dissipativity preserving model reduction by retention of trajectories of minimal dissipation. In Sect. 5, we turn to behaviors in driving variable representation, and characterize strict dissipativity in terms of the representation. We also establish a representation of the subbehavior of minimal dissipation in terms of the matrices of the driving variable representation. Using these results, in Sect. 6 we give an algorithm to solve our main problem (the problem introduced in Sect. 4) for the case that the behavior to be reduced is in driving variable representation. We also show that our reduced order behavior solves a Nevanlinna tangential interpolation problem. In Sect. 7, we give concluding remarks. Finally, in Appendix we review the necessary material on driving variable and output nulling representations and the way they interact.

In this paper, we will use the following notation: the space of n-dimensional real, respectively complex, vectors is denoted by $\mathbb{R}^{n}$, respectively $\mathbb{C}^{n}$, and the space of $m \times n$ real, respectively complex, matrices, by $\mathbb{R}^{\mathrm{m} \times \mathrm{n}}$, respectively $\mathbb{C}^{\mathrm{m} \times \mathrm{n}}$. Given two column vectors $x$ and $y$, we denote with $\operatorname{col}(x, y)$ the vector obtained by stacking $x$ over $y$; a similar convention holds for the stacking of matrices with the same number of columns. Given a Hermitian matrix $S \in \mathbb{C}^{\mathrm{w} \times \mathrm{w}}$, we define its inertia as the triple $\sigma(S):=$ $\left(\sigma_{-}, \sigma_{0}, \sigma_{+}\right)$where $\sigma_{+}$is the number of positive eigenvalues of $S, \sigma_{-}$is the number of negative eigenvalues of $S$, and $\sigma_{0}$ is the multiplicity of 0 as an eigenvalue of $S$.

The ring of polynomials with real coefficients in the indeterminate $\xi$ is denoted by $\mathbb{R}[\xi]$; the ring of two-variable polynomials with real coefficients in the indeterminates $\zeta$ and $\eta$ is denoted by $\mathbb{R}[\zeta, \eta]$. The space of all $\mathrm{m} \times \mathrm{n}$ polynomial matrices in the indeterminate $\xi$ is denoted by $\mathbb{R}^{\mathrm{m} \times \mathrm{n}}[\xi]$, and that consisting of all $\mathrm{m} \times \mathrm{n}$ polynomial matrices in the indeterminates $\zeta$ and $\eta$ by $\mathbb{R}^{\mathrm{m} \times \mathrm{n}}[\zeta, \eta]$. Given a matrix $R \in \mathbb{R}^{\mathrm{m} \times \mathrm{n}}[\xi]$, we define $R^{\sim}(\xi):=R(-\xi)^{T} \in \mathbb{R}^{\mathrm{n} \times \mathrm{m}}[\xi]$.

We denote with $\mathfrak{C}^{\infty}\left(\mathbb{R}, \mathbb{R}^{\mathrm{W}}\right)$ the set of infinitely often differentiable functions from $\mathbb{R}$ to $\mathbb{R}^{\mathrm{w}}$, and with $\mathfrak{D}\left(\mathbb{R}, \mathbb{R}^{\mathrm{w}}\right)$ the subspace of $\mathfrak{C}^{\infty}\left(\mathbb{R}, \mathbb{R}^{\mathrm{w}}\right)$ consisting of all compactly supported functions. We also denote with $\mathfrak{L}_{2}\left(\mathbb{R}, \mathbb{R}^{\mathrm{w}}\right)$ the set of all Lebesgue measurable functions $w$ from $\mathbb{R}$ to $\mathbb{R}^{\mathrm{w}}$ for which the integral $\int_{-\infty}^{\infty}\|w\|^{2} \mathrm{~d} t$ is finite, and with $\mathfrak{L}_{2}^{\text {loc }}\left(\mathbb{R}, \mathbb{R}^{\mathrm{w}}\right)$ the set of all Lebesgue measurable functions $w$ from $\mathbb{R}$ to $\mathbb{R}^{\mathrm{w}}$ for which the integral $\int_{\Omega}\|w\|^{2} \mathrm{~d} t$ is finite for all compact sets $\Omega \subset \mathbb{R}$. Sometimes, when the domain and co-domain are obvious from the context, we simply write $\mathfrak{C}^{\infty}, \mathfrak{D}, \mathfrak{L}_{2}$, and $\mathfrak{L}_{2}^{\text {loc }}$. Finally, if $F(t)$ is a real $\mathrm{p} \times \mathrm{m}$ matrix valued function, then the space of all functions formed as real linear combinations of the columns of $F(t)$ is denoted by $\operatorname{span}\{F(t)\}:=\left\{F(t) x_{0} \mid x_{0} \in \mathbb{R}^{m}\right\}$. 


\section{Behaviors and quadratic differential forms}

A subset $\mathfrak{B} \subset \mathfrak{C}^{\infty}\left(\mathbb{R}, \mathbb{R}^{\mathrm{W}}\right)$ is called a linear time-invariant differential system (briefly, a behavior ) if there exists a polynomial matrix $R \in \mathbb{R}^{\bullet \times w}[\xi]$ such that $\mathfrak{B}=\{w \in$ $\mathfrak{C}^{\infty}\left(\mathbb{R}, \mathbb{R}^{\mathrm{w}}\right) \mid R\left(\frac{\mathrm{d}}{\mathrm{d} t}\right) w=0$. By $\mathfrak{L}^{\mathrm{w}}$ we denote the set of all linear time-invariant differential systems with w variables. We note that while we define $\mathfrak{B} \in \mathfrak{L}^{\mathrm{w}}$ as the kernel of a differential operator, $\mathfrak{B}$ is often not specified in this way. We speak about a kernel representation when $\mathfrak{B} \in \mathfrak{L}^{\mathrm{w}}$ is represented by $R\left(\frac{\mathrm{d}}{\mathrm{d} t}\right) w=0$, i.e., $\mathfrak{B}=\{w \in$ $\left.\mathfrak{C}^{\infty}\left(\mathbb{R}, \mathbb{R}^{\mathrm{w}}\right) \mid R\left(\frac{\mathrm{d}}{\mathrm{d} t}\right) w=0\right\}$. Another representation is a latent variable representation, defined through polynomial matrices $R$ and $M$ by $R\left(\frac{\mathrm{d}}{\mathrm{d} t}\right) w=M\left(\frac{\mathrm{d}}{\mathrm{d} t}\right) \ell$, with $\mathfrak{B}=\left\{w \in \mathfrak{C}^{\infty}\left(\mathbb{R}, \mathbb{R}^{\mathrm{w}}\right) \mid \exists \ell \in \mathfrak{C}^{\infty}\left(\mathbb{R}, \mathbb{R}^{1}\right)\right.$ such that $\left.R\left(\frac{\mathrm{d}}{\mathrm{d} t}\right) w=M\left(\frac{\mathrm{d}}{\mathrm{d} t}\right) \ell\right\}$. This type of model is the kind of model that usually results from first principles modeling, with the $w$ 's the vector of variables that the model aims at, and the $\ell$ 's the vector of auxiliary variables introduced in the modeling process (for example state variables). The behavior $\mathfrak{B}$ is then called the external behavior, and $\mathfrak{B}_{\text {full }}=\left\{(w, \ell) \in \mathfrak{C}^{\infty}\left(\mathbb{R}, \mathbb{R}^{\mathrm{w} \times 1}\right) \mid\right.$ $\left.R\left(\frac{\mathrm{d}}{\mathrm{d} t}\right) w=M\left(\frac{\mathrm{d}}{\mathrm{d} t}\right) \ell\right\}$, the full behavior. If $\mathfrak{B}$ is the external behavior of $\mathfrak{B}_{\text {full }}$, then we often write $\mathfrak{B}=\left(\mathfrak{B}_{\text {full }}\right)_{\text {ext }}$.

We also need the notion of state for a behavior. We refer to [17] for a detailed exposition, with only a brief review here. A latent variable representation of $\mathfrak{B} \in \mathfrak{L}^{\mathrm{W}}$ is called a state representation if the latent variable (denoted here by $x$ ) has the property of state, i.e.: if $\left(w_{1}, x_{1}\right),\left(w_{2}, x_{2}\right) \in \mathfrak{B}_{\text {full }}$ are such that $x_{1}(0)=x_{2}(0)$ then $\left(w_{1}, x_{1}\right) \wedge\left(w_{2}, x_{2}\right)$, the concatenation (at $t=0$, here), belongs to the $\mathcal{L}_{2}^{\text {loc }}$-closure of $\mathfrak{B}_{\text {full }}$. We call such an $x$ a state for $\mathfrak{B}$. A given $\mathfrak{B} \in \mathfrak{L}^{\mathrm{w}}$ in general has many state representations. It turns out however that the minimal dimension of the state variable $x$ over all state representations only depends on the given behavior $\mathfrak{B}$. This number is called the McMillan degree of $\mathfrak{B}$, and is denoted by $n(\mathfrak{B})$. If $\mathfrak{B}$ is represented by $R\left(\frac{\mathrm{d}}{\mathrm{d} t}\right) w=0$, with $R$ a full row rank $\mathrm{p} \times$ w polynomial matrix, then $\mathrm{n}(\mathfrak{B})$ is equal to the maximal degree over all polynomials $\operatorname{det}\left(R_{1}\right)$, where $R_{1}$ ranges over all $\mathrm{p} \times \mathrm{p}$ minors of $R$, see [16].

A latent variable representation is a state representation of its manifest behavior if and only if its full behavior can be represented by a differential equation that is 0 th order in $w$ and first order in $x$, i.e., by $R_{0} w=M_{0} x+M_{1} \frac{\mathrm{d}}{\mathrm{d} t} x$, with $R_{0}, M_{0}, M_{1}$ constant matrices. There are many, more structured, state representations as, for instance, a driving variable representation $\frac{\mathrm{d}}{\mathrm{d} t} x=A x+B v, w=C x+D v$, with $v$ an, obviously free, additional latent variable; an output nulling representation $\frac{\mathrm{d}}{\mathrm{d} t} x=A x+B w, 0=C x+D w$; or an input/state/output representations $\frac{\mathrm{d}}{\mathrm{d} t} x=A x+B u, y=C x+D u, w=(u, y)$, the most popular of them all. Every system $\mathfrak{B} \in \mathfrak{L}^{\mathrm{w}}$ admits such a representation after a suitable permutation of the components of $w$ and a suitable choice of the state. In this paper, an important role is played by driving variable representations and output nulling representations. We have collected the basic material on these representations in Appendix.

In this paper, we restrict ourselves to controllable behaviors. In general, controllable behaviors are defined as behaviors in which for any two of its elements there exists a third element which coincides with the first one on the past and the second one on 
the future (for details, see [16]). $\mathfrak{L}_{\text {cont }}^{\mathrm{w}}\left(\right.$ a subset of $\mathfrak{L}^{\mathrm{w}}$ ) denotes the set of controllable behaviors.

Given a behavior $\mathfrak{B} \in \mathfrak{L}^{\mathrm{w}}$, it is in general possible to choose some components of $w$ as any function in $\mathfrak{C}^{\infty}(\mathbb{R}, \mathbb{R})$. The maximal number of such components that can be chosen arbitrarily is called the input cardinality of $\mathfrak{B}$ and is denoted as $m(\mathfrak{B})$. If none of the components of $w$ can be chosen arbitrarily, then $\mathfrak{B}$ is called autonomous, and we write $m(\mathfrak{B})=0$. The number $m(\mathfrak{B})$ is exactly equal to the dimension of the input $u$ in any input/state/output representation of $\mathfrak{B}$. The complementary number $w-m(\mathfrak{B})$ is called the output cardinality of $\mathfrak{B}$.

This paper also uses the formalism of quadratic differential form $(Q D F)$ developed in [30]. We now review the basic elements of the theory of QDF's. A two-variable polynomial matrix $\Phi \in \mathbb{R}^{\mathrm{w} \times \mathrm{w}}[\zeta, \eta]$ can be written as $\Phi(\zeta, \eta)=\sum_{h, k=0}^{N} \Phi_{h, k} \zeta^{h} \eta^{k}$, where $\Phi_{h, k} \in \mathbb{R}^{\mathrm{w} \times \mathrm{w}}$ for all $h, k$, and $N$ is a nonnegative integer. The two-variable polynomial matrix $\Phi(\zeta, \eta)$ induces a quadratic functional acting on w-dimensional infinitely differentiable trajectories, defined as $Q_{\Phi}(w)=\sum_{h, k=0}^{N}\left(\frac{\mathrm{d}^{h} w}{\mathrm{~d} t^{h}}\right)^{T} \Phi_{h, k} \frac{\mathrm{d}^{k} w}{\mathrm{~d} t^{k}}$. Such a functional is called a quadratic differential form (QDF). It is easy to see that without loss of generality we may restrict our attention to symmetric two-variable polynomial matrices $\Phi(\zeta, \eta)$, i.e. $\Phi(\zeta, \eta)=\Phi(\eta, \zeta)^{\top}$. In this paper we always assume that this is the case. By $\mathbb{R}_{s}^{\mathrm{W} \times \mathrm{W}}[\zeta, \eta]$ we will denote the subset of $\mathbb{R}^{\mathrm{W} \times \mathrm{w}}[\zeta, \eta]$ of all symmetric two-variable polynomial matrices.

\section{Dissipativity and the subbehavior of minimal dissipation}

For an extensive treatment of dissipative systems in a behavioral context we refer to $[21,27,30,31]$. Here, we review the basic material. Let $\Sigma=\Sigma^{\top} \in \mathbb{R}^{\mathrm{w} \times \mathrm{w}}$ and $\mathfrak{B} \in \mathfrak{L}_{\text {cont }}^{w}$. Write $Q_{\Sigma}(w):=w^{T} \Sigma w . \mathfrak{B}$ is said to be dissipative with respect to $Q_{\Sigma}$ (or briefly, $\Sigma$-dissipative) if $\int_{-\infty}^{+\infty} Q_{\Sigma}(w) \mathrm{d} t \geq 0$ for all $w \in \mathfrak{B} \cap \mathfrak{D}$. Further, it is said to be dissipative on $\mathbb{R}_{-}$with respect to $Q_{\Sigma}$ (or briefly, $\Sigma$-dissipative on $\mathbb{R}_{-}$) if $\int_{-\infty}^{0} Q_{\Sigma}(w) \mathrm{d} t \geq 0$ for all $w \in \mathfrak{B} \cap \mathfrak{D}$. We also use the analogous definition of dissipativity on $\mathbb{R}_{+}$. It is easily seen that if $\mathfrak{B}$ is $\Sigma$-dissipative on $\mathbb{R}_{-}$or $\mathbb{R}_{+}$, then it is $\Sigma$-dissipative. Identifying $Q_{\Sigma}(w)(t)$ with the rate of energy delivered to the system at time $t, \Sigma$-dissipativity states that the system absorbs energy when it is taken through any trajectory in $\mathfrak{B}$ that starts and ends with the system at rest. $\Sigma$-dissipativity on $\mathbb{R}_{-}$ is a stronger property which states (due to time invariance) that at any time the net flow of energy up to that time has already been into the system. Likewise, an interpretation can be given for $\Sigma$-dissipativity on $\mathbb{R}_{+}$. A controllable behavior $\mathfrak{B}$ is said to be strictly dissipative with respect to $Q_{\Sigma}$ (or briefly, strictly $\Sigma$-dissipative) if there exists an $\epsilon>0$ such that $\mathfrak{B}$ is dissipative with respect to $Q_{\Sigma-\epsilon I}$. We have the obvious definitions for strict dissipativity on $\mathbb{R}_{-}$and on $\mathbb{R}_{+}$. If $\mathfrak{B}$ is strictly $\Sigma$-dissipative on $\mathbb{R}_{-}$or $\mathbb{R}_{+}$, then it is strictly $\Sigma$-dissipative.

Remark 3.1 Note that these definitions of dissipativity are completely in terms of trajectories of the behavior $\mathfrak{B}$, and not in terms of any of its particular representations. For any particular type of representation the question can then be posed how to characterize dissipativity in terms of this representation. For example, if $\mathfrak{B}$ is given in 
image representation, $w=M\left(\frac{\mathrm{d}}{\mathrm{d} t}\right) \ell$, then it is $\Sigma$-dissipative if and only if $M^{\top}(-i \omega)$ $\Sigma M(i \omega) \geq 0$ for all $\omega \in \mathbb{R}$ (see [30]). From this, a signature condition for $\Sigma$-dissipativity can be derived for the case that $\mathfrak{B}$ is given in kernel representation. This is however beyond the scope of this paper. For systems represented in DV-representation conditions for (strict) $\Sigma$-dissipativity on $\mathbb{R}_{-}$and $\mathbb{R}_{+}$are given Propositions 5.2. if

The QDF $Q_{\Psi}$ induced by $\Psi \in \mathbb{R}_{s}^{\mathrm{w} \times \mathrm{w}}[\zeta, \eta]$ is called a storage function for $\left(\mathfrak{B}, Q_{\Sigma}\right)$

$$
\frac{\mathrm{d}}{\mathrm{d} t} Q_{\Psi}(w) \leq Q_{\Sigma}(w) \text { for all } w \in \mathfrak{B} \cap \mathfrak{C}^{\infty} .
$$

The QDF $Q_{\Delta}$ induced by $\Delta \in \mathbb{R}_{s}^{\mathrm{w} \times \mathrm{w}}[\zeta, \eta]$ is called a dissipation function for $\left(\mathfrak{B}, Q_{\Sigma}\right)$ if $Q_{\Delta}(w) \geq 0$ for all $w \in \mathfrak{B} \cap \mathfrak{C}^{\infty}$ and

$$
\int_{-\infty}^{\infty} Q_{\Sigma}(w) \mathrm{d} t=\int_{-\infty}^{\infty} Q_{\Delta}(w) \mathrm{d} t \text { for all } w \in \mathfrak{B} \cap \mathfrak{D}
$$

If the supply rate $Q_{\Sigma}$, the dissipation function $Q_{\Delta}$, and the storage function $Q_{\Psi}$ satisfy

$$
\frac{\mathrm{d}}{\mathrm{d} t} Q_{\Psi}(w)=Q_{\Sigma}(w)-Q_{\Delta}(w) \text { for all } w \in \mathfrak{B} \cap \mathfrak{C}^{\infty}
$$

then we call the triple $\left(Q_{\Sigma}, Q_{\Psi}, Q_{\Delta}\right)$ matched on $\mathfrak{B}$. Equation (2) expresses that, along $w \in \mathfrak{B}$, the increase in internal storage is equal to the rate at which supply is delivered minus the rate at which supply is dissipated. The following is well known, see e.g. [21].

\section{Proposition 3.2 The following conditions are equivalent}

1. $\left(\mathfrak{B}, Q_{\Sigma}\right)$ is dissipative,

2. ( $\left.\mathfrak{B}, Q_{\Sigma}\right)$ admits a storage function,

3. $\left(\mathfrak{B}, Q_{\Sigma}\right)$ admits a dissipation function.

Furthermore, for any dissipation function $Q_{\Delta}$ there exists a unique storage function $Q_{\Psi}$, and for any storage function $Q_{\Psi}$ there exists a unique dissipation function $Q_{\Delta}$ such that $\left(Q_{\Sigma}, Q_{\Psi}, Q_{\Delta}\right)$ is matched on $\mathfrak{B}$.

Now we introduce the notion of subbehavior of minimal dissipation. For a given $\Sigma$-dissipative system $\mathfrak{B}$, let $Q_{\Psi}$ be a storage function, and $Q_{\Delta}$ be a dissipation function such that $\left(Q_{\Sigma}, Q_{\Psi}, Q_{\Delta}\right)$ is matched on $\mathfrak{B}$. Let $w \in \mathfrak{B}$. Then the integral $\int_{t_{0}}^{t_{1}} Q_{\Delta}(w) \mathrm{d} t$ is equal to the dissipated supply over the interval $\left[t_{0}, t_{1}\right]$ when $\mathfrak{B}$ is taken through the trajectory $w$. It is tempting to state that for $w \in \mathfrak{B}$, over the whole real axis the amount of dissipated supply is equal to $\int_{-\infty}^{\infty} Q_{\Delta}(w) \mathrm{d} t$. However, in general this integral will not converge. Yet, we would like to consider trajectories $w \in \mathfrak{B}$ that are local minima of dissipation. Therefore we consider the change in dissipation if $w$ is compared to $w+\delta$, with $\delta \in \mathfrak{B}$ of compact support: Fix $w \in \mathfrak{B}$, and for $\delta \in \mathfrak{B} \cap \mathfrak{D}$ define 


$$
J_{w}(\delta):=\int_{-\infty}^{+\infty} Q_{\Delta}(w+\delta)-Q_{\Delta}(w) \mathrm{d} t
$$

Note that this integral always converges.

Definition 3.3 We call $w \in \mathfrak{B}$ a trajectory of minimal dissipation if $J_{w}(\delta) \geq 0$ for all $\delta \in \mathfrak{B} \cap \mathfrak{D}$, i.e if whenever $w \in \mathfrak{B}$ is perturbed to $w+\delta$ with $\delta \in \mathfrak{B}$ of compact support, there is no decrease in dissipated supply.

In a sense then, such $w$ 's are local minima of the amount of dissipated supply $\int_{-\infty}^{\infty} Q_{\Delta}$ $(w) \mathrm{d} t$, keeping in mind that the integral might not converge. In the sequel we show that there are many of these local minima, and that, together, they form a subbehavior of $\mathfrak{B}$. In fact, define

$$
\mathfrak{B}^{*}:=\{w \in \mathfrak{B} \mid \quad w \text { is a trajectory of minimal dissipation }\} .
$$

It turns out that the subbehavior $\mathfrak{B}^{*}$ of $\mathfrak{B}$ of trajectories of minimal dissipation is independent of the chosen dissipation function $Q_{\Delta}$, forms a behavior again, and admits an easy characterization in terms of $\mathfrak{B}$ and $\Sigma$. For this, define the $\Sigma$-orthogonal complement $\mathfrak{B}^{\perp_{\Sigma}}$ of $\mathfrak{B}$ as

$$
\mathfrak{B}^{\perp_{\Sigma}}:=\left\{w \in \mathfrak{C}^{\infty} \mid \int_{-\infty}^{+\infty} w^{\top} \Sigma \delta \mathrm{d} t=0 \text { for all } \delta \in \mathfrak{B} \cap \mathfrak{D}\right\}
$$

It can be proven that $\mathfrak{B}^{\perp_{\Sigma}}$ is also a controllable behavior, see Section 10 of [30]. If $\Sigma=I$, we simply write $\mathfrak{B}^{\perp}$, called the orthogonal complement of $\mathfrak{B}$. We then have:

Theorem 3.4 Let $\mathfrak{B} \in \mathfrak{L}_{\text {contr }}^{\mathrm{w}}$ and $\Sigma=\Sigma^{\top} \in \mathbb{R}^{\mathrm{w} \times \mathrm{w}}$. Assume that $\mathfrak{B}$ is $\Sigma$-dissipative. Then $\mathfrak{B}^{*} \in \mathfrak{L}^{\mathrm{w}}$ and

$$
\mathfrak{B}^{*}=\mathfrak{B} \cap \mathfrak{B}^{\perp \Sigma}=\mathfrak{B} \cap(\Sigma \mathfrak{B})^{\perp} .
$$

Proof Let $Q_{\Delta}$ be a dissipation function. Let $Q_{\Psi}$ a storage function such that $\frac{\mathrm{d}}{\mathrm{d} t} Q_{\Psi}=$ $Q_{\Sigma}-Q_{\Delta}$. It is then easily seen that for all $w \in \mathfrak{B}$ and for all $\delta \in \mathfrak{B} \cap \mathfrak{D}$ we have

$$
J_{w}(\delta)=\int_{-\infty}^{+\infty} Q_{\Sigma}(w+\delta)-Q_{\Sigma}(w) \mathrm{d} t
$$

Clearly, $J_{w}(\delta)=\int_{-\infty}^{+\infty} \delta^{\top} \Sigma \delta \mathrm{d} t+2 \int_{-\infty}^{+\infty} w^{\top} \Sigma \delta \mathrm{d} t$, and it can be seen that $J_{w}(\delta) \geq 0$ for all $\delta \in \mathfrak{B} \cap \mathfrak{D}$ if and only if the linear term is equal to zero for all $\delta \in \mathfrak{B} \cap \mathfrak{D}$. Consequently, the subset of trajectories $\mathfrak{B}$ of minimal dissipation is equal to 


$$
\mathfrak{B}^{*}=\left\{w \in \mathfrak{B} \mid \int_{-\infty}^{+\infty} w^{\top} \Sigma \delta \mathrm{d} t=0 \text { for all } \delta \in \mathfrak{B} \cap \mathfrak{D}\right\}=\mathfrak{B} \cap(\mathfrak{B})^{\perp_{\Sigma}}
$$

The subbehavior $\mathfrak{B}^{*}$ plays an important role in the sequel:

Definition 3.5 Let $\mathfrak{B} \in \mathfrak{L}_{\text {contr }}^{\mathrm{w}}$ and $\Sigma=\Sigma^{\top} \in \mathbb{R}^{\mathrm{w} \times \mathrm{w}}$. Assume that $\mathfrak{B}$ is $\Sigma$-dissipative. $\mathfrak{B}^{*}$ as defined by (3) is called the subbehavior of minimal dissipation.

It turns out that if $\mathfrak{B}$ is strictly dissipative, then the subbehavior $\mathfrak{B}^{*}$ of minimal dissipation is autonomous. In fact, we have

Theorem 3.6 Assume that $\mathfrak{B} \in \mathfrak{L}_{\text {contr }}^{\mathrm{W}}$ is strictly $\Sigma$-dissipative. Then

1. $\mathfrak{B}^{*}$ is autonomous,

2. $\mathfrak{B}^{*}=\left(\mathfrak{B}^{*}\right)_{\text {stab }} \oplus\left(\mathfrak{B}^{*}\right)_{\text {antistab}}$, where we define $\left(\mathfrak{B}^{*}\right)_{\text {stab }}:=\left\{w \in \mathfrak{B}^{*} \mid \lim _{t \rightarrow \infty}\right.$ $w(t)=0\}$ and $\left(\mathfrak{B}^{*}\right)_{\text {antistab }}:=\left\{w \in \mathfrak{B}^{*} \mid \lim _{t \rightarrow-\infty} w(t)=0\right\}$,

3. $\mathrm{n}\left(\mathfrak{B}^{*}\right)=2 \mathrm{n}(\mathfrak{B})$ and $\mathrm{n}\left(\left(\mathfrak{B}^{*}\right)_{\text {stab }}\right)=\mathrm{n}\left(\left(\mathfrak{B}^{*}\right)_{\text {antistab }}\right)=\mathrm{n}(\mathfrak{B})$.

Proof A proof of this follows immediately from Lemma 5.4 (and the remarks following it) in Sect. 5.

If $\mathfrak{B}^{*}$ is autonomous there exists a square, nonsingular polynomial matrix, say $\bar{R}(\xi)$ such that $\mathfrak{B}^{*}$ is represented by $\bar{R}\left(\frac{\mathrm{d}}{\mathrm{d} t}\right) w=0$, see [16]. The roots of the polynomial $\operatorname{det}(\bar{R})$ do not depend on the particular representation, since any two representations $\bar{R}$ and $\bar{R}^{\prime}$, square and nonsingular, are related via a unimodular matrix: $\bar{R}=U \bar{R}^{\prime}$ (see [16]).

Definition 3.7 Let $\bar{R}(\xi)$ be a square, nonsingular polynomial matrix such that $\mathfrak{B}^{*}$ is represented by $\bar{R}\left(\frac{\mathrm{d}}{\mathrm{d} t}\right) w=0$. Then the roots of the polynomial $\operatorname{det}(\bar{R})$ are called the spectral zeros of $\mathfrak{B}$.

Clearly, these complex numbers coincide with the characteristic values that appear in the exponential parts of the trajectories in $\mathfrak{B}^{*}$.

Remark 3.8 In Lemma 5.4 of this paper, a state space representations for $\mathfrak{B}^{*}$ will be obtained for the case that the original behavior $\mathfrak{B}$ is given in DV-representation. In Example 3.9 below, $\mathfrak{B}^{*}$ is computed for the case that $\mathfrak{B}$ is given in input-state-output representation. If $\mathfrak{B}$ is given in image representation, $w=M\left(\frac{\mathrm{d}}{\mathrm{d} t}\right) \ell$, then a representation of $\mathfrak{B}^{*}$ is given by $w=M\left(\frac{\mathrm{d}}{\mathrm{d} t}\right) \ell, M^{\top}\left(-\frac{\mathrm{d}}{\mathrm{d} t}\right) \Sigma M\left(\frac{\mathrm{d}}{\mathrm{d} t}\right) \ell=0$. The spectral zeros are the roots of the polynomial matrix $M^{\top}(-\xi) \Sigma M(\xi)$. If $\mathfrak{B}$ is given in kernel representation $R\left(\frac{\mathrm{d}}{\mathrm{d} t}\right) w=0$ and $\Sigma$ is invertible, then it can be shown that a representation of $\mathfrak{B}^{*}$ is given by $w=\Sigma^{-1} R^{\top}\left(-\frac{\mathrm{d}}{\mathrm{d} t}\right) \ell, R\left(\frac{\mathrm{d}}{\mathrm{d} t}\right) \Sigma^{-1} R^{\top}\left(-\frac{\mathrm{d}}{\mathrm{d} t}\right) \ell=0$. This also shows that the spectral zeros of $\mathfrak{B}$ are the roots of the polynomial matrix $R(\xi) \Sigma^{-1} R^{\top}(-\xi)$. The stable part of $\mathfrak{B}^{*}$ is then represented by $w=\Sigma^{-1} R^{\top}\left(-\frac{\mathrm{d}}{\mathrm{d} t}\right) \ell, H\left(\frac{\mathrm{d}}{\mathrm{d} t}\right) \ell=0$, where $R(\xi) \Sigma^{-1} R^{\top}(-\xi)=A(\xi) H(\xi)$ is a polynomial factorization with $A$ anti-Hurwitz and $H$ Hurwitz (this can be obtained using spectral factorization). The antistable part is obtained analogously. Since these result are not used in this paper, the details are omitted. 
Example 3.9 In order to connect our work with that of Sorensen [19], as an extended example we consider the class of strictly passive input-output systems. Let $A \in \mathbb{R}^{\mathrm{n} \times \mathrm{n}}$, $B \in \mathbb{R}^{\mathrm{n} \times \mathrm{u}}, C \in \mathbb{R}^{\mathrm{Y} \times \mathrm{n}}, D \in \mathbb{R}^{\mathrm{Y} \times \mathrm{u}}$, with $A$ stable, $(A, B)$ a controllable pair and $(C, A)$ an observable pair, and consider the equations

$$
\begin{aligned}
& \dot{x}=A x+B u, \\
& y=C x+D u .
\end{aligned}
$$

Let $\mathfrak{B} \in \mathfrak{L}_{\text {contr }}^{\mathrm{u}+\mathrm{y}}$ be the external behavior corresponding to these equations, i.e.,

$$
\mathfrak{B}=\left\{(u, y) \in \mathfrak{C}^{\infty}\left(\mathbb{R}, \mathbb{R}^{\mathrm{u}+\mathrm{y}}\right) \mid \text { there exists } x \in \mathfrak{C}^{\infty}\left(\mathbb{R}, \mathbb{R}^{\mathrm{n}}\right) \text { such that (4) holds }\right\}
$$

Assume the system is strictly passive, i.e., there exists $\epsilon>0$ such that $\int_{-\infty}^{t} u(s)^{\top} y(s)$ $\mathrm{d} s \geq \epsilon \int_{-\infty}^{t} u(s)^{\top} u(s) \mathrm{d} s$ for all $t \in \mathbb{R}$ and all $(u, y) \in \mathfrak{B} \cap \mathfrak{L}_{2}\left(\mathbb{R}, \mathbb{R}^{\mathrm{u}+\mathrm{y}}\right)$. It is easily verified that this property is equivalent to $\mathfrak{B}$ being strictly $\Sigma$-dissipative on $\mathbb{R}_{-}$, with $\Sigma$ given by

$$
\Sigma=\frac{1}{2}\left[\begin{array}{cc}
0 & I_{\mathrm{u}} \\
I_{\mathrm{u}} & 0
\end{array}\right]
$$

By [31], Section VI, $\mathfrak{B}^{\perp \Sigma}$ is equal to the external behavior corresponding to the equations

$$
\begin{aligned}
& \dot{z}=-A^{\top} z+C^{\top} u, \\
& y=B^{\top} z-D^{\top} u .
\end{aligned}
$$

The subbehavior of minimal dissipation $\mathfrak{B}^{*}=\mathfrak{B} \cap \mathfrak{B}^{\perp \Sigma}$ is thus equal to the external behavior of the behavior represented by the combined equations (4) and 5). Subtracting the two equations for $y$, we get $C x-B^{\top} z+\left(D+D^{\top}\right) u=0$. By strict passivity we have that $D+D^{\top}>0$. Thus $u=-\left(D+D^{\top}\right)^{-1} C x+\left(D+D^{\top}\right)^{-1} B^{\top} z$, so $\mathfrak{B}^{*}$ is equal to the external behavior corresponding to the equations

$$
\left[\begin{array}{c}
\dot{x} \\
\dot{z}
\end{array}\right]=H\left[\begin{array}{l}
x \\
z
\end{array}\right],\left[\begin{array}{l}
u \\
y
\end{array}\right]=L\left[\begin{array}{l}
x \\
z
\end{array}\right]
$$

where we define $H$ to be the Hamiltonian matrix

$$
H:=\left[\begin{array}{ll}
A-B\left(D+D^{\top}\right)^{-1} C & B\left(D+D^{\top}\right)^{-1} B^{\top} \\
-C^{\top}\left(D+D^{\top}\right)^{-1} C & -\left(A-B\left(D+D^{\top}\right)^{-1} C\right)^{\top}
\end{array}\right]
$$

and $L$ in the output of equation (6) by

$$
L:=\left[\begin{array}{ll}
-\left(D+D^{\top}\right)^{-1} C & \left(D+D^{\top}\right)^{-1} B^{\top} \\
C-D\left(D+D^{\top}\right)^{-1} C & D\left(D+D^{\top}\right)^{-1} B^{\top}
\end{array}\right]
$$


Note that, indeed, $\mathfrak{B}^{*}$ is autonomous, and its dynamics is governed by the Hamiltonian matrix $H$ of (7). The eigenvalues of $H$ coincide with the spectral zeros (see Definition 3.7) of the system $\mathfrak{B}$. In turn these coincide with the zeros of $G(\xi)+G^{\top}(-\xi)$, where $G(\xi)=D+C(\xi I-A)^{-1} B$. The stable part $\left(\mathfrak{B}^{*}\right)_{\text {stab }}$ is obtained from (6) as follows: let $X$ and $Y$ be real $\mathrm{n} \times \mathrm{n}$ matrices such that

$$
H\left[\begin{array}{l}
X \\
Y
\end{array}\right]=\left[\begin{array}{l}
X \\
Y
\end{array}\right] R_{-}
$$

with $R_{-}$an $\mathrm{n} \times \mathrm{n}$ matrix such that $\operatorname{Re}(\lambda)<0$ for all $\lambda \in \sigma\left(R_{-}\right)$. Then

$$
\left(\mathfrak{B}^{*}\right)_{\text {stab }}=\left\{\left[\begin{array}{l}
u \\
y
\end{array}\right] \mid \exists \xi \in \mathbb{R}^{\mathrm{n}} \text { such that }\left[\begin{array}{c}
u(t) \\
y(t)
\end{array}\right]=L\left[\begin{array}{c}
X \\
Y
\end{array}\right] \mathrm{e}^{R_{-} t} \xi\right\} .
$$

Likewise, the antistable part $\left(\mathfrak{B}^{*}\right)_{\text {antistab }}$ is obtained by computing the antistable (n-dimensional) invariant subspace of $H$ (note: $H$ has no eigenvalues on the imaginary axis due to strict dissipativity). We will return to this example in Sect. 4.

Remark 3.10 Another important special case is the case of strictly bounded real inputoutput systems, in which case $\Sigma$ is given $\Sigma=\operatorname{diag}\left(-I_{\mathrm{u}}, I_{\mathrm{y}}\right)$. Similar computations as in Example 3.9 can be given in this case. The spectral zeros then coincide with the zeros of $G^{\top}(-\xi) G(\xi)-I$, where $G(\xi)=D+C(\xi I-A)^{-1} B$.

\section{Problem statement}

In this section, we will formulate the problem of model reduction by retention of trajectories of minimal dissipation.

Main Problem. Let $\mathfrak{B} \in \mathfrak{L}_{\text {contr }}^{\mathrm{w}}$. Let $\Sigma=\Sigma^{\top} \in \mathbb{R}^{\mathrm{w} \times \mathrm{w}}$. Assume that $\mathfrak{B}$ is strictly $\Sigma$-dissipative on $\mathbb{R}^{-}$. Let $\left(\mathfrak{B}^{*}\right)$ antistable be the antistable part of the subbehavior of minimal dissipation $\mathfrak{B}^{*}$. Let $\mathrm{k}<\mathrm{n}(\mathfrak{B})$ be given together with a subbehavior $\mathfrak{B}^{\prime} \subset$ $\left(\mathfrak{B}^{*}\right)_{\text {antistable }}$ such that $n\left(\mathfrak{B}^{\prime}\right)=k$. Find $\hat{\mathfrak{B}} \in \mathfrak{L}_{\text {contr }}^{\mathrm{w}}$ such that

1. $\mathrm{n}(\hat{\mathfrak{B}}) \leq \mathrm{k}$,

2. $\mathrm{m}(\hat{\mathfrak{B}})=\mathrm{m}(\mathfrak{B})$,

3. $\hat{\mathfrak{B}}$ is strictly dissipative on $\mathbb{R}^{-}$with respect to $Q_{\Sigma}$,

4. The antistable part $\left(\hat{\mathfrak{B}}^{*}\right)_{\text {antistable }}$ of $\hat{\mathfrak{B}}^{*}$ is a subbehavior of $\mathfrak{B}^{\prime}$.

Any behavior $\hat{\mathfrak{B}}$ as above has the property that the $\hat{\mathbf{n}}$-dimensional antistable part of the subbehavior of minimal dissipation (with $\hat{n}=n(\hat{\mathfrak{B}})$ ) is contained in the antistable part of the subbehavior of minimal dissipation of the original system $\mathfrak{B}$. Thus, $\hat{\mathfrak{B}}$ inherits from $\mathfrak{B}$ a $\hat{n}$-dimensional subbehavior of its subbehavior of minimal dissipation. By virtue of this property, $\hat{\mathfrak{B}}$ is considered as an approximation of $\mathfrak{B}$. Note that there are many choices for the $\mathrm{k}$-dimensional subbehavior $\mathfrak{B}^{\prime}$ of $\left(\mathfrak{B}^{*}\right)$ antistable. Different choices of $\mathfrak{B}^{\prime}$ will of course result in different approximations $\hat{\mathfrak{B}}$. Note that the given behavior $\mathfrak{B}^{\prime}$, as a subbehavior of the autonomous behavior $\mathfrak{B}^{*}$, is autonomous. It does therefore itself not qualify as a solution to our problem: property (2) does not 
hold, and property (3) cannot hold since dissipativity is only defined for controllable systems.

In the sequel we will prove that the subbehavior of minimal dissipation $\mathfrak{B}^{*}$ is associated with the spectral zeros of the original system. This already appeared to be the case in Example 3.9. It will turn out that any of the approximants $\hat{\mathfrak{B}}$ that we will obtain as solution to our problem allows an interpretation as a solution of a rational interpolation problem, with as interpolation points some of the antistable spectral zeros together with their mirror images in the imaginary axis, see also [2] and [11].

Of course, it is also possible to formulate a version of the Main Problem with strict dissipativity on $\mathbb{R}^{+}$, and with $\mathfrak{B}^{\prime}$ a subbehavior of the stable part of $\mathfrak{B}^{*}$, in which the problem is to find a reduced order behavior $\hat{\mathfrak{B}}$ such that $\hat{\mathfrak{B}}^{*}$ is a subbehavior of the stable part of $\hat{\mathfrak{B}}^{*}$. The details are left to the reader.

Next, we continue with Example 3.9, and show that the method of Sorensen [19] solves our Main Problem for the special case of strictly passive input-output systems.

Example 4.1 In order to obtain a strictly passive reduced order model with state space dimension $\mathrm{k}<\mathrm{n}$, in [19] a $\mathrm{k}$-dimensional $H$-invariant subspace of the $\mathrm{n}$-dimensional antistable subspace of $H$ given by (7) is computed. In particular, [19] computes real $\mathrm{n} \times \mathrm{k}$ matrices $X, Y$, and a real $\mathrm{k} \times \mathrm{k}$ matrix $R$ with $\operatorname{Re}(\lambda)>0$ for all $\lambda \in \sigma(R)$ such that

$$
H\left[\begin{array}{l}
X \\
Y
\end{array}\right]=\left[\begin{array}{l}
X \\
Y
\end{array}\right] R
$$

It is proven in [19] that $X$ and $Y$ have full column rank, and that $X^{\top} Y$ is symmetric. In addition, since the system is strictly passive, $X^{\top} Y$ is positive definite (note that in this paper, we use the opposite sign convention of [19]). Next, let $X^{\top} Y=Q S^{2} Q^{\top}$ be a spectral decomposition, with $Q^{\top} Q=Q Q^{\top}=I$ and $S$ a real diagonal matrix with positive diagonal elements, and put $V=X Q S^{-1}, W=Y Q S^{-1}$. Next, define the reduced order system $\hat{\mathfrak{B}}$ as the external behavior of

$$
\begin{aligned}
\dot{x_{1}} & =\hat{A} x_{1}+\hat{B} u, \\
y & =\hat{C} x_{1}+\hat{D} u .
\end{aligned}
$$

with $(\hat{A}, \hat{B}, \hat{C}, \hat{D}):=\left(W^{\top} A V, W^{\top} B, C V, D\right)$. Sorensen proves in [19] that this reduced order system is stable and strictly passive again. We will now explain how this relates to our Main Problem.

The k-dimensional $H$-invariant subspace im $\left[\begin{array}{c}X \\ Y\end{array}\right]$ corresponds to a unique k-dimensional subbehavior $\mathfrak{B}^{\prime}$ of the $n$-dimensional antistable part $\left(\mathfrak{B}^{*}\right)_{\text {antistab }}$, namely

$$
\mathfrak{B}^{\prime}=\left\{\left[\begin{array}{l}
u \\
y
\end{array}\right] \mid \exists \xi \in \mathbb{R}^{\mathrm{k}} \quad \text { such that }\left[\begin{array}{c}
u(t) \\
y(t)
\end{array}\right]=L\left[\begin{array}{c}
X \\
Y
\end{array}\right] \mathrm{e}^{R t} \xi\right\}
$$

We prove that $\mathfrak{B}^{\prime}$ is equal to the antistable part $\left(\hat{\mathfrak{B}}^{*}\right)_{\text {antistable }}$ of the reduced order system $\hat{\mathfrak{B}}$, so that, in addition to the first three conditions, also Condition 4 of our Main 
Problem is satisfied by the reduced order behavior $\hat{\mathfrak{B}}$. Observe that $\hat{\mathfrak{B}}^{*}$ is equal to the external behavior corresponding to the equations

$$
\left[\begin{array}{c}
\dot{x}_{1} \\
\dot{z}_{1}
\end{array}\right]=\hat{H}\left[\begin{array}{l}
x_{1} \\
z_{1}
\end{array}\right],\left[\begin{array}{l}
u \\
y
\end{array}\right]=\hat{L}\left[\begin{array}{l}
x_{1} \\
z_{1}
\end{array}\right]
$$

with $\hat{H}$ and $\hat{L}$ defined as in (7) and (8), with $(A, B, C, D)$ replaced by $(\hat{A}, \hat{B}, \hat{C}, \hat{D})$. Following [19], define $\mathrm{k} \times \mathrm{k}$ matrices $\hat{X}$ and $\hat{Y}$ by $\hat{X}=\hat{Y}=S Q^{\top}$. It is then easily verified that

$$
\hat{H}\left[\begin{array}{l}
\hat{X} \\
\hat{Y}
\end{array}\right]=\left[\begin{array}{l}
\hat{X} \\
\hat{Y}
\end{array}\right] R
$$

so $\operatorname{im}\left[\begin{array}{c}\hat{X} \\ \hat{Y}\end{array}\right]$ is equal to the $\mathrm{k}$-dimensional antistable subspace of the reduced Hamiltonian $\hat{H}$. Thus the antistable part of $\hat{\mathfrak{B}}^{*}$ must be equal to

$$
\left(\hat{\mathfrak{B}}^{*}\right)_{\text {antistable }}=\left\{\left[\begin{array}{l}
u \\
y
\end{array}\right] \mid \exists \xi \in \mathbb{R}^{\mathrm{k}} \text { such that }\left[\begin{array}{c}
u(t) \\
y(t)
\end{array}\right]=\hat{L}\left[\begin{array}{c}
\hat{X} \\
\hat{Y}
\end{array}\right] \mathrm{e}^{R t} \xi\right\} .
$$

Since, by construction $\hat{L} \hat{X}=L X$ and $\hat{L} \hat{Y}=L Y$, the latter is indeed equal to $\mathfrak{B}^{\prime}$ given by (11). Thus we have shown that the method of Sorensen computes for the given $\mathrm{k}$-dimensional subbehavior $\mathfrak{B}^{\prime}$ (corresponding to his k-dimensional $H$-invariant subspace $\operatorname{im}\left[\begin{array}{l}X \\ Y\end{array}\right]$ ) a reduced order system $\hat{\mathfrak{B}}$ that satisfies the four conditions of our Main Problem. This concludes the example.

In formulating the problem of model reduction by retention of trajectories of minimal dissipation, we have kept with one of the tenets of behavioral systems theory, that of articulating concepts at the most intrinsic possible level, that of trajectories. In practice, though, the to-be-approximated behavior $\mathfrak{B}$ is represented in some form, be it kernel, image, latent variable, state space, etc., and the issue arises of how to pass from the original representation to a representation of a reduced order approximation, for example for the purposes of simulation, of control, etc. In the remainder of this paper we consider this topic for only one type of model, namely driving-variable (in the following abbreviated with DV), and delay the discussion of other types of representations to Sect.7, where we outline some of the lines of research currently pursued. The definitions of DV and output nulling (ON) representations, and some of the essential notions necessary in order to understand the material presented in this paper, are gathered in Appendix.

\section{Dissipativity and minimal dissipation for DV representations}

In this section, we examine strict dissipativity and the subbehavior of minimal dissipation for the case that our system is represented by a DV-representation. 
The connection between dissipativity, the algebraic Riccati equation (ARE), and the Hamiltonian matrix of the system is well known, see [27-29,32]. In the following, we will review this connection for the case of half-line dissipativity. First note the following:

Lemma 5.1 Let $\Sigma=\Sigma^{\top} \in \mathbb{R}^{\mathrm{w} \times \mathrm{w}}$. Let $\mathfrak{B} \in \mathfrak{L}_{\mathrm{contr}}^{\mathrm{w}}$ be strictly $\Sigma$-dissipative. Then there exists a minimal driving variable representation $\mathfrak{B}_{\mathrm{DV}}(A, B, C, D)$ of $\mathfrak{B}$ with $D^{\top} \Sigma D=I$ and $D^{\top} \Sigma C=0$.

Proof Let $\hat{A}, \hat{B}, \hat{C}, \hat{D}$ be such that $\mathfrak{B}_{\mathrm{DV}}(\hat{A}, \hat{B}, \hat{C}, \hat{D})$ is a minimal DV representation of $\mathfrak{B}$. Then $\hat{D}$ has full column rank (see Appendix, Proposition 8.1). We prove now that $\hat{D}^{\top} \Sigma \hat{D}>0$. Take the driving variable $v(t)=\sqrt{\delta(t)} v_{0}$, with $\delta(t)$ representing the Dirac pulse. Then, with state trajectory $x(t)=0$ for all $t \in \mathbb{R}, \dot{x}=A x+B v$ holds, so $w(t)=\hat{D} v(t)$. There exists $\epsilon>0$ such that

$$
\begin{aligned}
v_{0}^{\top} \hat{D}^{\top} \Sigma \hat{D} v_{0} & =\int_{-\infty}^{\infty} v(t)^{\top} \hat{D}^{\top} \Sigma \hat{D} v(t) \mathrm{d} t=\int_{-\infty}^{\infty} w(t)^{\top} \Sigma w(t) \mathrm{d} t \geq \epsilon \int_{-\infty}^{\infty} w(t)^{\top} w(t) \mathrm{d} t \\
& \geq \epsilon v_{0}^{\top} \hat{D}^{\top} \hat{D} v_{0} .
\end{aligned}
$$

Of course, a rigorous proof can be given using smooth approximations of $\delta$. This proves the claim. Let $W$ be a nonsingular matrix such that $\hat{D}^{\top} \Sigma \hat{D}=W^{\top} W$. By applying the state feedback transformation $\hat{v}=-\left(\hat{D}^{\top} \Sigma \hat{D}\right)^{-1} \hat{D}^{\top} \Sigma \hat{C} x+W^{-1} v$ to $\mathfrak{B}_{\mathrm{DV}}(\hat{A}, \hat{B}, \hat{C}, \hat{D})$ we obtain a new driving variable representation $\mathfrak{B}_{\mathrm{DV}}(A, B, C, D)$ of $\mathfrak{B}$, with

$$
\begin{aligned}
A & =\hat{A}-\hat{B}\left(\hat{D}^{\top} \Sigma \hat{D}\right)^{-1} \hat{D}^{\top} \Sigma \hat{C}, \\
B & =\hat{B} W^{-1}, \\
C & =\hat{C}-\hat{D}\left(\hat{D}^{\top} \Sigma \hat{D}\right)^{-1} \hat{D}^{\top} \Sigma \hat{C}, \\
D & =\hat{D} W^{-1} .
\end{aligned}
$$

Observe that $D$ is injective, and that from the minimality of $\mathfrak{B}_{\mathrm{DV}}(\hat{A}, \hat{B}, \hat{C}, \hat{D})$ and statement (2) of Proposition 8.1 it follows that $\mathfrak{B}_{\mathrm{DV}}(A, B, C, D)$ is also a minimal representation of $\mathfrak{B}$. It is easy to see that $D^{\top} \Sigma D=I$, and moreover

$$
D^{\top} \Sigma C=W^{-\top} \hat{D}^{\top} \Sigma\left(\hat{C}-\hat{D}\left(\hat{D}^{\top} \Sigma \hat{D}\right)^{-1} \hat{D}^{\top} \Sigma \hat{C}\right)=0
$$

This concludes the proof.

We then have the following:

Proposition 5.2 Let $\mathfrak{B} \in \mathfrak{L}_{\text {contr }}^{\mathrm{w}}$, and let $\Sigma=\Sigma^{\top} \in \mathbb{R}^{\mathrm{w} \times \mathrm{w}}$. Let $\mathfrak{B}_{\mathrm{DV}}(A, B, C, D)$ be a minimal driving variable representation of $\mathfrak{B}$ such that $D^{\top} \Sigma D=I$ and $D^{\top} \Sigma C=0$. If $\mathfrak{B}$ is strictly $\Sigma$-dissipative then the Hamiltonian matrix

$$
H=\left[\begin{array}{cc}
A & B B^{\top} \\
C^{\top} \Sigma C & -A^{\top}
\end{array}\right]
$$


has no eigenvalues on the imaginary axis. Furthermore, the following statements are equivalent:

1. $\mathfrak{B}$ is strictly $\Sigma$-dissipative on $\mathbb{R}^{-}\left(\mathbb{R}^{+}\right)$,

2. the ARE

$$
A^{\top} K+K A-C^{\top} \Sigma C+K B B^{\top} K=0
$$

has a real symmetric solution $K$ with $K>0(K<0)$ and $A+B B^{\top} K$ is antistable (stable),

3. The Hamiltonian matrix (13) has no eigenvalues on the imaginary axis, and there exists $X_{1}, Y_{1} \in \mathbb{R}^{\mathrm{n} \times \mathrm{n}}$, with $X_{1}$ nonsingular, and $M \in \mathbb{R}^{\mathrm{n} \times \mathrm{n}}$ antistable (stable) such that

$$
H\left[\begin{array}{c}
X_{1} \\
Y_{1}
\end{array}\right]=\left[\begin{array}{c}
X_{1} \\
Y_{1}
\end{array}\right] M
$$

with $X_{1}^{\top} Y_{1}>0\left(X_{1}^{\top} Y_{1}<0\right)$.

If $K$ satisfies the conditions in (2.) above then it is unique, and it is the largest (smallest) real symmetric solution of (14). We denote it by $K^{+}\left(K^{-}\right)$. If $X_{1}, Y_{1}$ satisfy the conditions in (3.) above, then $Y_{1} X_{1}^{-1}$ is equal to this largest (smallest) real symmetric solution $K^{+}\left(K^{-}\right)$of the ARE (14).

Proof Assume that $H$ has an eigenvalue $i \omega$, with eigenvector $\left(x_{1}^{*}, x_{2}^{*}\right)^{*}$. Then $A x_{1}+$ $B B^{\top} x_{2}=i \omega x_{1}$ and $C^{\top} \Sigma C x_{1}-A^{\top} x_{2}=0$. We will first prove that the vector

$$
w_{0}:=C x_{1}+D B^{\top} x_{2}
$$

is unequal to 0 . Indeed, assume $w_{0}=0$. Then premultiplying with $D^{\top} \Sigma$ yields $B^{\top} x_{2}=0$. This yields $A x_{1}=i \omega x_{1}$ and $C x_{1}=0$. By observability of the pair $(C, A)$ we then obtain $x_{1}=0$. Thus $\left(i \omega I+A^{\top}\right) x_{2}=0$, whence $x_{2}^{*}(A-i \omega I)=0$. Together with $x_{2}^{*} B=0$, by controllability of the pair $(A, B)$ this yields also $x_{2}=0$. Consequently, $w_{0} \neq 0$. It is also easily verified that $w_{0}^{*} \Sigma w_{0}=0$.

Let $\Delta>0$. Consider the differential equation $\dot{x}=A x+B v$. Using controllability of the pair $(A, B)$, let $\tilde{v}_{1}:(-\infty, 0] \rightarrow \mathbb{R}^{\mathrm{V}}$ be a driving variable trajectory that drives state 0 at $t=-\Delta$ to state $x_{1}$ at time $t=0$. Choose $\tilde{v}_{1}$ such that $\tilde{v}_{1}(t)=0$ for $t<-\Delta$. Let $\tilde{x}_{1}(t)(t \leq 0)$ be the corresponding state trajectory, and $\tilde{w}_{1}(t):=C \tilde{x}_{1}+D \tilde{v}_{1}(t)$. Likewise, let $\tilde{v}_{2}:[0, \infty) \rightarrow \mathbb{R}^{\mathrm{V}}$ be a driving variable trajectory that drives state $x_{1}$ at $t=0$ to state 0 at time $t=\Delta$. Choose $\tilde{v}_{2}$ such that $\tilde{v}_{2}(t)=0$ for $t>\Delta$. Let $\tilde{x}_{2}(t)$ $(t \geq 0)$ be the corresponding state trajectory, and $\tilde{w}_{2}(t):=C \tilde{x}_{2}+D \tilde{v}_{2}(t)$.

Denote $T=\frac{2 \pi}{\omega}$ and define for our driving variable representation $\mathfrak{B}_{\mathrm{DV}}(A, B, C, D)$ a sequence of driving variable trajectories $v_{n}$ by

$$
v_{n}(t)= \begin{cases}\tilde{v}_{1}(t+n T) & t<-n T, \\ \mathrm{e}^{i \omega t} B^{\top} x_{2} & -n T \leq t<n T, \\ \tilde{v}_{2}(t-n T) & t \geq n T .\end{cases}
$$


Define

$$
x_{n}(t)= \begin{cases}\tilde{x}_{1}(t+n T) & t<-n T, \\ \mathrm{e}^{i \omega t} x_{1} & -n T \leq t<n T, \\ \tilde{x}_{2}(t-n T) & t \geq n T .\end{cases}
$$

Then $\dot{x}_{n}(t)=A x_{n}(t)+B v_{n}(t)$ for all $t \in \mathbb{R}$. Also, for $t \in(-\infty,-n T-\Delta] \cup$ $[n T+\Delta, \infty)$ we have $x_{n}(t)=0$, so $w_{n}(t)=0$. For $t \in(-n T-\Delta,-n T]$ we have $w_{n}(t)=\tilde{w}_{1}(t+n T)$, for $t \in(-n T, n T)$ we have $w_{n}(t)=w_{0} \mathrm{e}^{i \omega t}$ [with $w_{0}$ given by (15)], and for $t \in[n T, n T+\Delta)$ we have $w_{n}(t)=\tilde{w}_{2}(t-n T)$. In particular, for any $n, w_{n}$ has compact support.

Now, clearly, $\int_{-\infty}^{\infty}\left|w_{n}(t)\right|^{2} \mathrm{~d} t \rightarrow \infty$ as $n \rightarrow \infty$. On the other hand however, $w_{n}(t)^{*} \Sigma w_{n}(t)=w_{0}^{*} \Sigma w_{0}=0$ for $t \in(-n T, n T)$, so $\int_{-\infty}^{\infty} w_{n}(t)^{*} \Sigma w_{n}(t) \mathrm{d} t=$ $\int_{-\Delta}^{0} \tilde{w}_{1}(t) \mathrm{d} t+\int_{0}^{\Delta} \tilde{w}_{2}(t) \mathrm{d} t$, independent of $n$. Thus, for $n$ sufficiently large the inequality $\int_{-\infty}^{\infty} w_{n}(t)^{*} \Sigma w_{n}(t) \mathrm{d} t \geq \epsilon \int_{-\infty}^{\infty}\left|w_{n}(t)\right|^{2} \mathrm{~d} t$ fails to hold. Now, $w_{n}$ is of course not in $\mathfrak{D}$. However, $\mathfrak{D}$ is dense in $\mathfrak{L}_{2}$, and by approximating $v_{n}$ by driving variable trajectories in $\mathfrak{D}$ we can obtain a smooth $w_{n}$, contradicting the assumption that our system is strictly $\Sigma$-dissipative. This proves that $H$ has no eigenvalues on the imaginary axis.

(1) $\Longrightarrow$ (2). In both cases, $\mathfrak{B}$ is strictly $\Sigma$-dissipative, so the Hamiltonian $H$ has no eigenvalues on the imaginary axis. It then follows from standard results on the Hamiltonian matrix, using controllability of $(A, B)$, (see e.g. [32]) that the ARE (14) has a real symmetric solution $K$ such that $A+B B^{\top} K$ is antistable, and also a real symmetric solution $K$ such that $A+B B^{\top} K$ is stable. It was proven in [12], Theorem 5.3.4 that if $\mathfrak{B}$ is strictly $\Sigma$-dissipative on $\mathbb{R}_{-}$then the antistabilizing solution $K$ is positive definite. In a similar way it can be proven that if $\mathfrak{B}$ is strictly $\Sigma$-dissipative on $\mathbb{R}_{+}$then the stabilizing solution $K$ is negative definite.

(2) $\Longrightarrow$ (1). This was also proven in [12], Theorem 5.3.4.

(2) $\Longleftrightarrow$ (3). This equivalence follows from standard results on the relation between the algebraic Riccati equation and Hamiltonian matrices, see e.g. [32].

Remark 5.3 It can be shown (see [20]) that any real symmetric solution $K$ of the ARE (14) yields a storage function $Q_{\Psi}(w)=x^{\top} K x$ (with $x$ the unique trajectory state trajectory corresponding to $w \in \mathfrak{B}$ ). Moreover, the smallest (largest) storage function is $x^{\top} K_{-} x\left(x^{\top} K_{+} x\right)$. The unique dissipation function corresponding to the storage function $x^{\top} K x$ is $Q_{\Delta}(w)=\left\|B^{\top} K x-v\right\|^{2}$.

In the remainder of this section, for systems represented in DV form we will obtain a representation of the subbehavior of minimal dissipation, and of its antistable and stable part.

From Proposition 8.6 in Appendix it follows that if $\mathfrak{B}_{\mathrm{DV}}(A, B, C, D)$ is a minimal driving variable representation of $\mathfrak{B} \in \mathfrak{L}_{\mathrm{contr}}^{\mathrm{w}}$, then $\mathfrak{B}_{\mathrm{ON}}\left(-A^{\top}, C^{\top} \Sigma, B^{\top},-D^{\top} \Sigma\right)$ is a minimal output nulling representation of $\mathfrak{B}^{\perp \Sigma}$. Using Theorem 3.4 we then find that if $\mathfrak{B}$ is $\Sigma$-dissipative, then the subbehavior of minimal dissipation of $\mathfrak{B}$ is given by

$$
\mathfrak{B}^{*}=\mathfrak{B}_{\mathrm{DV}}(A, B, C, D)_{\mathrm{ext}} \cap \mathfrak{B}_{\mathrm{ON}}\left(-A^{\top}, C^{\top} \Sigma, B^{\top},-D^{\top} \Sigma\right)_{\mathrm{ext}} .
$$


For strictly $\Sigma$-dissipative systems this yields the following state space representation of $\mathfrak{B}^{*}$ :

Lemma 5.4 Let $\mathfrak{B} \in \mathfrak{L}_{\mathrm{contr}}^{\mathrm{w}}$ be strictly $\Sigma$-dissipative, and let $\mathfrak{B}_{\mathrm{DV}}(A, B, C, D)$ be a minimal $D V$ representation of $\mathfrak{B}$ such that $D^{\top} \Sigma D=I$ and $D^{\top} \Sigma C=0$. Then $\mathfrak{B}^{*}$ is equal to the external behavior of

$$
\begin{aligned}
{\left[\begin{array}{c}
\dot{x} \\
\dot{z}
\end{array}\right] } & =\left[\begin{array}{ll}
A & B B^{\top} \\
C^{\top} \Sigma C & -A^{\top}
\end{array}\right]\left[\begin{array}{l}
x \\
z
\end{array}\right] \\
w & =\left[\begin{array}{ll}
C & D B^{\top}
\end{array}\right]\left[\begin{array}{l}
x \\
z
\end{array}\right],
\end{aligned}
$$

i.e., $\mathfrak{B}^{*}=\left\{w \in \mathfrak{C}^{\infty}\left(\mathbb{R}, \mathbb{R}^{\mathrm{w}}\right) \mid\right.$ there exist $x, z \in \mathfrak{C}^{\infty}\left(\mathbb{R}, \mathbb{R}^{\mathrm{n}}\right)$ such that (17) holds $\}$.

Proof By (21), $w \in \mathfrak{B}^{*}$ if and only if there exist $x, z, v$ such that

$$
\begin{aligned}
\dot{x} & =A x+B v, \\
\dot{z} & =-A^{\top} z+C^{\top} \Sigma w, \\
w & =C x+D v, \\
0 & =B^{\top} z-D^{\top} \Sigma w .
\end{aligned}
$$

Since $D^{\top} \Sigma D=I$ and $D^{\top} \Sigma C=0$, from (18) and (19) it follows that $B^{\top} z=$ $D^{\top} \Sigma w=D^{\top} \Sigma(C x+D v)=v$. Also, $\dot{z}=-A^{\top} z+C^{\top} \Sigma(C x+D v)=-A^{\top} z+$ $C^{\top} \Sigma C x$. This proves the claim of the lemma.

As a consequence, the spectral zeros of $\mathfrak{B}$ coincide with the eigenvalues of the Hamiltonian matrix $H$ (13). In turn, these can be shown to coincide with the zeros of the proper rational matrix $G^{\top}(-\xi) \Sigma G(\xi)$, with $G(\xi):=C(\xi I-A)^{-1} B+D$.

The full behavior represented by the Equations (17) will be called the Hamiltonian behavior of $\mathfrak{B}_{\mathrm{DV}}(A, B, C, D)$ with respect to $\Sigma$, and we denote it with $\mathfrak{B}_{H}(A, B, C, D)$. Thus, $\mathfrak{B}^{*}$ is equal to the external behavior $\mathfrak{B}_{H}(A, B, C, D)$ ext of the Hamiltonian behavior. Clearly, the antistable (stable) part of the external behavior of (17) can be obtained by considering the antistable (stable) invariant subspace $X_{+}(H)\left(X_{-}(H)\right)$ of the Hamiltonian matrix $H$. Indeed, since (by strict $\Sigma$-dissipativity) $H$ has no imaginary axis eigenvalues, if $X_{1}, Y_{1} \in \mathbb{R}^{\mathrm{n} \times \mathrm{n}}$ are such that the columns of $\operatorname{col}\left(X_{1}, Y_{1}\right)$ form a basis of $X_{+}(H)$, and $M \in \mathbb{R}^{\mathrm{n} \times \mathrm{n}}$ is the matrix of $\left.H\right|_{X_{+}(H)}$ with respect to this basis, then

$$
\left(\mathfrak{B}^{*}\right)_{\text {antistab }}=\operatorname{span}\left\{\left(C X_{1}+D B^{\top} Y_{1}\right) \mathrm{e}^{M t}\right\}
$$

Now suppose a subbehavior $\mathfrak{B}^{\prime}$ of $\mathfrak{B}^{*}$ is given, with $\mathrm{n}\left(\mathfrak{B}^{\prime}\right)=\mathrm{k}$. It can be shown that any such $\mathfrak{B}^{\prime}$ corresponds to a unique k-dimensional $H$-invariant subspace of $\mathbb{R}^{2 \mathrm{n}}$, the state space of $\mathfrak{B}^{*}$. Thus, we obtain the following:

Theorem 5.5 Let $\mathfrak{B} \in \mathfrak{L}_{\text {contr }}^{\mathrm{w}}$ be strictly $\Sigma$-dissipative on $\mathbb{R}^{-}$, and let $\mathfrak{B}_{\mathrm{DV}}(A, B$, $C, D)$ be a minimal $D V$ representation of $\mathfrak{B}$, with $D^{\top} \Sigma D=I$ and $D^{\top} \Sigma C=0$. 
Let $\mathrm{k}<\mathrm{n}(\mathfrak{B})$ be a positive integer. Let $\mathfrak{B}^{\prime}$ be a subbehavior of $\left(\mathfrak{B}^{*}\right)_{\text {antistab with }}$ $\mathrm{n}\left(\mathfrak{B}^{\prime}\right)=\mathrm{k}$. Then there exist $X_{1}^{1}, Y_{1}^{1} \in \mathbb{R}^{\mathrm{n} \times \mathrm{k}}, X_{1}^{2}, Y_{1}^{2} \in \mathbb{R}^{\mathrm{n} \times(\mathrm{n}-\mathrm{k})}$, and matrices $M_{11}, M_{12}, M_{22}$ with $M_{11}$ and $M_{22}$ antistable and $X_{1}:=\left[X_{1}^{1} X_{1}^{2}\right]$ nonsingular, such that

$$
\begin{aligned}
& {\left[\begin{array}{cc}
A & B B^{\top} \\
C^{\top} \Sigma C & -A^{\top}
\end{array}\right]\left[\begin{array}{cc}
X_{1}^{1} & X_{1}^{2} \\
Y_{1}^{1} & Y_{1}^{2}
\end{array}\right]=\left[\begin{array}{cc}
X_{1}^{1} & X_{1}^{2} \\
Y_{1}^{1} & Y_{1}^{2}
\end{array}\right] \underbrace{\left[\begin{array}{ll}
M_{11} & M_{12} \\
0 & M_{22}
\end{array}\right]}_{=: M},} \\
& \mathfrak{B}^{\prime}=\operatorname{span}\left\{\left(C X_{1}^{1}+D B^{\top} Y_{1}^{1}\right) \mathrm{e}^{M_{11} t}\right\},
\end{aligned}
$$

and

$$
\left(\mathfrak{B}^{*}\right)_{\text {antistab }}=\operatorname{span}\left\{\left(C X_{1}+D B^{\top} Y_{1}\right) e^{M t}\right\} .
$$

Here, we define $Y_{1}:=\left[\begin{array}{ll}Y_{1}^{1} & Y_{1}^{2}\end{array}\right]$.

Proof A proof follows immediately from Lemma 5.4 and the remarks above.

A similar theorem of course holds for the stable part $\left(\mathfrak{B}^{*}\right)_{\text {stable }}$ of $\mathfrak{B}^{*}$ under the assumption of strict $\Sigma$-dissipativity on $\mathbb{R}^{+}$.

To conclude this section we formulate a result that will be of importance in our reduction procedure. The result deals with a general, possibly non-controllable behavior, represented by a minimal DV-representation. It states that the subbehavior of minimal dissipation of the controllable part of $\mathfrak{B}$ is contained in the external behavior of the Hamiltonian system (17):

Lemma 5.6 Let $\mathfrak{B} \in \mathfrak{L}^{\mathrm{w}}$ and $\Sigma=\Sigma^{\top} \in \mathbb{R}^{\mathrm{w} \times \mathrm{w}}$. Let $\mathfrak{B}_{\mathrm{DV}}(A, B, C, D)$ be a minimal driving variable representation of $\mathfrak{B}$ such that $D^{\top} \Sigma D=I$ and $D^{\top} \Sigma C=0$. Assume that $\mathfrak{B}_{\text {contr }}$ is strictly $\Sigma$-dissipative. Then $\left(\mathfrak{B}_{\text {contr }}\right)^{*} \subseteq \mathfrak{B}_{H}(A, B, C, D)_{\text {ext }}$. Consequently, $\left(\left(\mathfrak{B}_{\text {contr }}\right)^{*}\right)_{\text {antistable }}$ is contained in the antistable part of $\mathfrak{B}_{H}(A, B, C, D)_{\text {ext }}$.

Proof Starting from the DV-representation $\mathfrak{B}_{\mathrm{DV}}(A, B, C, D)$ we first compute a minimal driving variable representation of the controllable part of $\mathfrak{B}$. In order to do this, we first compute a driving variable representation $\mathfrak{B}_{\mathrm{DV}}\left(\bar{A}_{11}, \bar{B}_{1}, \bar{C}_{1}, \bar{D}\right)$ of $\mathfrak{B}_{\text {contr }}$ following Proposition 8.4. Observe that this is in general not a minimal representation of $\mathfrak{B}_{\text {contr }}$. Therefore, we apply the feedback transformation $v=\bar{F} x+v^{\prime}$ with $\bar{F}:=$ $-\left(\bar{D}^{\top} \bar{D}\right)^{-1} \bar{D}^{\top} \bar{C}_{1}$, and a state space transformation $S$ to $\mathfrak{B}_{\mathrm{DV}}\left(\bar{A}_{11}, \bar{B}_{1}, \bar{C}_{1}, \bar{D}\right)$ (see Proposition 8.2), in order to obtain a minimal DV-representation of $\mathfrak{B}_{\text {contr }}: S^{-1}\left(\bar{A}_{11}+\right.$ $\left.\bar{B}_{1} \bar{F}\right) S=\left[\begin{array}{cc}\tilde{A}_{11} & 0 \\ \tilde{A}_{21} & \tilde{A}_{22}\end{array}\right], S^{-1} \bar{B}_{1}=\left[\begin{array}{c}\tilde{B}_{1} \\ \tilde{B}_{2}\end{array}\right],\left(\bar{C}_{1}+\bar{D} \bar{F}\right) S=\left[\begin{array}{cc}\tilde{C}_{1} & 0\end{array}\right], \tilde{D}=\bar{D}$. Then $\mathfrak{B}_{\mathrm{DV}}\left(\tilde{A}_{11}, \tilde{B}_{1}, \tilde{C}_{1}, \tilde{D}\right)$ is a minimal DV-representation of $\mathfrak{B}_{\text {contr. }}$ By Theorem 3.4 we have

$$
\begin{aligned}
\left(\mathfrak{B}_{\text {contr }}\right)^{*} & =\mathfrak{B}_{\text {contr }} \cap\left(\mathfrak{B}_{\text {contr }}\right)^{\perp_{\Sigma}} \\
& =\mathfrak{B}_{\text {contr }} \cap\left(\mathfrak{B}_{\mathrm{DV}}\left(\tilde{A}_{11}, \tilde{B}_{1}, \tilde{C}_{1}, \tilde{D}\right)_{\text {ext }}\right)^{\perp_{\Sigma}} .
\end{aligned}
$$


By minimality of the DV-representation, applying Proposition 8.6 we have

$$
\left(\mathfrak{B}_{\mathrm{DV}}\left(\tilde{A}_{11}, \tilde{B}_{1}, \tilde{C}_{1}, \tilde{D}\right)_{\mathrm{ext}}\right)^{\perp_{\Sigma}}=\mathfrak{B}_{\mathrm{ON}}\left(-\tilde{A}_{11}^{\top}, \tilde{C}_{1}^{\top} \Sigma, \tilde{B}_{1}^{\top},-\tilde{D}^{\top} \Sigma\right)_{\mathrm{ext}}
$$

Now observe that

$$
\begin{aligned}
\mathfrak{B}_{\mathrm{ON}}\left(-\tilde{A}_{11}^{\top}, \tilde{C}_{1}^{\top} \Sigma, \tilde{B}_{1}^{\top},-\tilde{D}^{\top} \Sigma\right)_{\mathrm{ext}} & \subseteq \mathfrak{B}_{\mathrm{ON}}\left(-\bar{A}_{11}^{\top}, \bar{C}_{1}^{\top} \Sigma, \bar{B}_{1}^{\top},-\bar{D}^{\top} \Sigma\right)_{\mathrm{ext}} \\
& \subseteq \mathfrak{B}_{\mathrm{ON}}\left(-A^{\top}, C^{\top} \Sigma, B^{\top},-D^{\top} \Sigma\right)_{\mathrm{ext}} .
\end{aligned}
$$

We will prove the first of the above inclusions. A proof of the second inclusion can be given in an analogous way. Let $w \in \mathfrak{B}_{\mathrm{ON}}\left(-\tilde{A}_{11}^{\top}, \tilde{C}_{1}^{\top} \Sigma, \tilde{B}_{1}^{\top},-\tilde{D}^{\top} \Sigma\right)_{\text {ext }}$. Then there exists $z_{1}$ such that $\dot{z}_{1}=-\tilde{A}_{11}^{\top} z_{1}+\tilde{C}_{1}^{\top} \Sigma w, 0=\tilde{B}_{1}^{\top} z_{1}-\tilde{D}^{\top} \Sigma w$. Define $z_{2}$ by $z_{2}(t)=0$ for all $t \in \mathbb{R}$, and put $x:=S\left[\begin{array}{l}z_{1} \\ z_{2}\end{array}\right]$. Then it can be verified that $x$ and $w$ satisfy the equations $\dot{x}=-\left(\bar{A}_{11}+\bar{B}_{1} \bar{F}\right)^{\top} x+\left(\bar{C}_{1}+\bar{D} \bar{F}\right)^{\top} \Sigma w, 0=\bar{B}_{1}^{\top} x-\bar{D}^{\top} \Sigma w$, equivalently, $\dot{x}=-\bar{A}_{11}^{\top} x+\bar{C}_{1}^{\top} \Sigma w, \quad 0=\bar{B}_{1}^{\top} x-\bar{D}^{\top} \Sigma w$. This implies that $w \in$ $\mathfrak{B}_{\mathrm{ON}}\left(-\bar{A}_{11}^{\top}, \bar{C}_{1}^{\top} \Sigma, \bar{B}_{1}^{\top},-\bar{D}^{\top} \Sigma\right)_{\mathrm{ext}}$, proving the inclusion.

Next, note that

$$
\mathfrak{B}_{\text {contr }} \subset \mathfrak{B}=\mathfrak{B}_{\mathrm{DV}}(A, B, C, D)_{\mathrm{ext}} .
$$

Combining (21), (22), (23) and (24), we obtain

$$
\begin{aligned}
\left(\mathfrak{B}_{\text {contr }}\right)^{*} & \subseteq \mathfrak{B}_{\mathrm{DV}}(A, B, C, D)_{\mathrm{ext}} \cap \mathfrak{B}_{\mathrm{ON}}\left(-A^{\top}, C^{\top} \Sigma, B^{\top},-D^{\top} \Sigma\right)_{\mathrm{ext}} \\
& =\mathfrak{B}_{H}(A, B, C, D)_{\mathrm{ext}} .
\end{aligned}
$$

The inclusion of the antistable parts then follows immediately. This concludes the proof of the lemma.

\section{A reduction algorithm for DV-representations}

In this section, we give an algorithmic procedure to compute for a given controllable behavior $\mathfrak{B}$, strictly $\Sigma$-dissipative on $\mathbb{R}^{-}$, a given integer $\mathrm{k} \leq \mathrm{n}(\mathfrak{B})$, and a given McMillan degree $\mathrm{k}$ subbehavior of the antistable part of the subbehavior of minimal dissipation, a DV-representation of a solution to our Main Problem as stated in Sect. 4. Subsequently, we will show that the transfer matrix from driving variable to manifest variable of any of our solutions is a solution to a rational interpolation problem associated with the data of the model reduction problem.

Algorithm 1 (from DVR to DVR)

Input: $\mathfrak{B} \in \mathfrak{L}_{\text {contr }}^{\mathrm{W}}$ strictly $\Sigma$-dissipative on $\mathbb{R}^{-}$, an integer $0 \leq \mathrm{k} \leq \mathrm{n}(\mathfrak{B})$ and a subbehavior $\mathfrak{B}^{\prime}$ of $\left(\mathfrak{B}^{*}\right)$ antistable of McMillan degree $\mathrm{k}$.

Output: A minimal DV-representation of $\hat{\mathfrak{B}} \in \mathfrak{L}_{\text {contr }}^{\mathrm{w}}$ satisfying the requirements of the Main Problem. 
Step 1. Represent $\mathfrak{B}$ by a minimal DV-representation $\mathfrak{B}_{\mathrm{DV}}(A, B, C, D)$ such that $D^{\top} \Sigma D=I$ and $D^{\top} \Sigma C=0$.

Step 2. Compute $X_{1}=\left[X_{1}^{1} X_{1}^{2}\right] \in \mathbb{R}^{\mathrm{n} \times \mathrm{n}}$ nonsingular, $Y_{1}=\left[Y_{1}^{1} Y_{1}^{2}\right] \in \mathbb{R}^{\mathrm{n} \times \mathrm{n}}$ such that

$$
\left[\begin{array}{cc}
A & B B^{\top} \\
C^{\top} \Sigma C & -A^{\top}
\end{array}\right]\left[\begin{array}{ll}
X_{1}^{1} & X_{1}^{2} \\
Y_{1}^{1} & Y_{1}^{2}
\end{array}\right]=\left[\begin{array}{cc}
X_{1}^{1} & X_{1}^{2} \\
Y_{1}^{1} & Y_{1}^{2}
\end{array}\right]\left[\begin{array}{cc}
M_{11} & M_{12} \\
0 & M_{22}
\end{array}\right],
$$

where $M_{11}$ and $M_{22}$ are antistable and $\mathfrak{B}^{\prime}=\operatorname{span}\left\{\left(C X_{1}^{1}+D B^{\top} Y_{1}^{1}\right) \mathrm{e}^{M_{11} t}\right\}$.

Step 3. Compute a Cholesky factorization $P^{\top} P=X_{1}^{\top} Y_{1},(P$ is a nonsingular upper triangular matrix).

Comment: Such factorization exists, since $\mathfrak{B} \in \mathfrak{L}_{\text {contr }}^{\mathrm{w}}$ is strictly $\Sigma$-dissipative on $\mathbb{R}^{-}$, so $X_{1}^{\top} Y_{1}$ is symmetric and positive definite, see Proposition 5.2. This also implies that $Y_{1}$ is nonsingular. Note that in general in a Cholesky factorization the diagonal elements are understood to be positive. Positivity of the diagonal elements will however not be used in the sequel, and only nonsingularity and the upper triangular structure are relevant).

Step 4. Define $S=X_{1} P^{-1}=Y_{1}^{-\top} P^{\top}$.

Step 5. Compute $(\bar{A}, \bar{B}, \bar{C}, \bar{D})=\left(S^{-1} A S, S^{-1} B, C S, D\right)$.

Step 6. Denote the truncation of $(\bar{A}, \bar{B}, \bar{C}, \bar{D})$ to the first $\mathrm{k}$ components of the state vector by $\left(\bar{A}_{11}, \bar{B}_{1}, \bar{C}_{1}, \bar{D}\right)$.

Step 7. Perform a Kalman controllability decomposition:

$$
T^{-1} \bar{A}_{11} T=\left[\begin{array}{c}
\hat{A} * \\
0 *
\end{array}\right], T^{-1} \bar{B}_{1}=\left[\begin{array}{l}
\hat{B} \\
0
\end{array}\right], \bar{C}_{1} T=[\hat{C} *], \bar{D}=\hat{D} .
$$

Step 8. Output

$$
\hat{\mathfrak{B}}:=\mathfrak{B}_{\mathrm{DV}}(\hat{A}, \hat{B}, \hat{C}, \hat{D})_{\mathrm{ext}}
$$

Proposition 6.1 The behavior $\hat{\mathfrak{B}}$ computed by Algorithm 1 is a solution to the Main Problem as formulated in Sect. 4.

Proof By construction $\mathrm{n}(\hat{\mathfrak{B}}) \leq \mathrm{k}$. Also, $D=\hat{D}$ has full column rank, so the number of driving variable components in the original and new DV-representation are equal. Since the number of driving variable components of a minimal DV-representation is equal to the input cardinality of its external behavior, we obtain $m(\hat{\mathfrak{B}})=m(\mathfrak{B})$.

We now prove that $\hat{\mathfrak{B}}$ is strictly $\Sigma$-dissipative on $\mathbb{R}^{-}$. It is easily verified that for $(\bar{A}, \bar{B}, \bar{C}, \bar{D})$ as computed in Step 5 above we have

$$
\left[\begin{array}{cc}
\bar{A} & \bar{B} \bar{B}^{\top} \\
\bar{C}^{\top} \Sigma \bar{C} & -\bar{A}^{\top}
\end{array}\right]\left[\begin{array}{l}
P \\
P
\end{array}\right]=\left[\begin{array}{c}
P \\
P
\end{array}\right] M, \quad \text { with } M=\left[\begin{array}{cc}
M_{11} & M_{12} \\
0 & M_{22}
\end{array}\right]
$$


as in Step 2. Denote the $(1,1)$-block of the upper triangular matrix $P$ by $P_{11}$. Then the truncated system $\left(\bar{A}_{11}, \bar{B}_{1}, \bar{C}_{1}, \bar{D}\right)$ computed in Step 6 satisfies

$$
\left[\begin{array}{cc}
\bar{A}_{11} & \bar{B}_{1} \bar{B}_{1}^{\top} \\
\bar{C}_{1}^{\top} \Sigma \bar{C}_{1}-\bar{A}_{11}^{\top}
\end{array}\right]\left[\begin{array}{l}
P_{11} \\
P_{11}
\end{array}\right]=\left[\begin{array}{l}
P_{11} \\
P_{11}
\end{array}\right] M_{11} .
$$

From (25) it then follows that the maximal solution of the ARE

$$
\bar{A}_{11}^{\top} \bar{K}+\bar{K} \bar{A}_{11}-\bar{C}_{1}^{\top} \Sigma \bar{C}_{1}+\bar{K} \bar{B}_{1} \bar{B}_{1}^{\top} \bar{K}=0
$$

is given by $\bar{K}^{+}=P_{11} P_{11}^{-1}=I$, the $\mathrm{k} \times \mathrm{k}$ identity matrix. Moreover, from (25) we also obtain $\left(\bar{A}_{11}+\bar{B}_{1} \bar{B}_{1}^{\top}\right) P_{11}=P_{11} M_{11}$, which implies that $\bar{A}_{11}+\bar{B}_{1} \bar{B}_{1}^{\top}$ is similar to $M_{11}$ and therefore antistable.

Now consider the ARE corresponding to the DV-representation of the reduced order (controllable) behavior $\hat{\mathfrak{B}}$ computed in Step 7:

$$
\hat{A}^{\top} \hat{K}+\hat{K} \hat{A}-\hat{C}^{\top} \Sigma \hat{C}+\hat{K} \hat{B} \hat{B}^{\top} \hat{K}=0
$$

and observe that any solution of $(27)$ is the $(1,1)$-block of a solution of (26). In particular, $\hat{K}=I$ (where $I$ is the $\mathrm{k}_{1} \times \mathrm{k}_{1}$ identity matrix, with $\mathrm{k}_{1}$ the size of $\hat{A}$ ) is a solution of (27). We claim that this solution $\hat{K}=I$ is antistabilizing, in the sense that $\hat{A}+\hat{B} \hat{B}^{\top} \hat{K}=\hat{A}+\hat{B} \hat{B}^{\top}$ is antistable. Indeed,

$$
\bar{A}_{11}+\bar{B}_{1} \bar{B}_{1}^{\top}=\left[\begin{array}{ll}
\hat{A}+\hat{B} \hat{B}^{\top} * \\
0 & *
\end{array}\right]
$$

As noted above, the left-hand side is antistable, so indeed $\hat{A}+\hat{B} \hat{B}^{\top}$ is antistable as well. Since $\hat{K}=I>0$, by Proposition 5.2 we conclude that $\hat{\mathfrak{B}}$ is strictly $\Sigma$-dissipative on $\mathbb{R}^{-}$.

We finally prove that the antistable part of the subbehavior of minimal dissipation of the reduced order behavior $\hat{\mathfrak{B}}$ is contained in $\mathfrak{B}^{\prime}$. In order to do so, first observe that

$$
\begin{aligned}
\mathfrak{B}^{\prime} & =\operatorname{span}\left\{\left(C X_{1}^{1}+D B^{\top} Y_{1}^{1}\right) \mathrm{e}^{M_{11} t}\right\} \\
& =\operatorname{span}\left\{\left((C S)\left(S^{-1} X_{1}^{1}\right)+\left(D B^{\top} S^{-\top}\right)\left(S^{\top} Y_{1}^{1}\right)\right) \mathrm{e}^{M_{11} t}\right\} \\
& =\operatorname{span}\left\{\left(\bar{C}\left(S^{-1} X_{1}^{1}\right)+\bar{D} \bar{B}^{\top}\left(S^{\top} Y_{1}^{1}\right)\right) \mathrm{e}^{M_{11} t}\right\} \\
& =\operatorname{span}\left\{\left[\bar{C}_{1} \bar{C}_{2}\right]\left[\begin{array}{c}
P_{11} \\
0
\end{array}\right] \mathrm{e}^{M_{11} t}+\left[\begin{array}{ll}
\bar{D} \bar{B}_{1}^{\top} & \bar{D} \bar{B}_{2}^{\top}
\end{array}\right]\left[\begin{array}{c}
P_{11} \\
0
\end{array}\right] \mathrm{e}^{M_{11} t}\right\} \\
& =\operatorname{span}\left\{\left(\bar{C}_{1}+\bar{D} \bar{B}_{1}^{\top}\right) P_{11} \mathrm{e}^{M_{11} t}\right\} .
\end{aligned}
$$

Note that the external behavior $\mathfrak{B}_{\text {trunc }}:=\mathfrak{B}_{\mathrm{DV}}\left(\bar{A}_{11}, \bar{B}_{1}, \bar{C}_{1}, \bar{D}\right)_{\text {ext }}$ may not be controllable, but that we do have $\bar{D}^{\top} \Sigma \bar{D}=I$ and $\bar{D}^{\top} \Sigma \bar{C}_{1}=0$. By applying Lemma 5.6 
we have

$$
\hat{\mathfrak{B}}^{*}=\left(\left(\mathfrak{B}_{\text {trunc }}\right)_{\text {contr }}\right)^{*} \subseteq \mathfrak{B}_{H}\left(\bar{A}_{11}, \bar{B}_{1}, \bar{C}_{1}, \bar{D}\right)_{\mathrm{ext}} .
$$

This implies that the antistable part $\left(\hat{\mathfrak{B}}^{*}\right)$ antistable must be contained in the antistable part of $\mathfrak{B}_{H}\left(\bar{A}_{11}, \bar{B}_{1}, \bar{C}_{1}, \bar{D}\right)_{\text {ext }}$. Using (25), the latter is equal to $\operatorname{span}\left\{\left(\bar{C}_{1}+\right.\right.$ $\left.\left.\bar{D} \bar{B}_{1}^{\top}\right) P_{11} \mathrm{e}^{M_{11} t}\right\}=\mathfrak{B}^{\prime}$. This concludes the proof.

Remark 6.2 Algorithm 1 constructs $a$ solution to our Main Problem. This solution is not unique, since even the construction of the algorithm does not lead to a unique solution. Indeed, starting with a DV-representation in Step 1, the matrices $X_{1}$ and $Y_{1}$ in Step 2 are of course not unique. Different choices of $X_{1}$ and $Y_{1}$ will lead to different Cholesky factors in Step 3, to different state space transformations in Step 4, so to different truncated systems in Step 6.

Remark 6.3 We note that our Algorithm 1 deals with DV-representations, whereas Sorensen's algorithm (see also Examples 3.9, 4.1) deals with input-state-output representations. Both algorithms compute suitable eigenspaces of the Hamiltonian matrix associated with the representation. However, whereas Sorensen's algorithm uses projections to arrive at a reduced order system, our Algorithm 1 computes a reduced order system by truncating the DV-representation obtained after a suitable state space transformation.

Remark 6.4 Algorithm 1 is of course also applicable to systems $\mathfrak{B}$ in input-stateoutput representation $\dot{x}=A x+B u, y=C x+D u$ that are either strictly passive or strictly bounded real. We will outline now how this can be done:

1. The strictly passive case: First transform the input-state-output representation for $\mathfrak{B}$ into a DV-representation $\dot{x}=A x+B v,\left[\begin{array}{l}u \\ y\end{array}\right]=\left[\begin{array}{l}0 \\ C\end{array}\right] x+\left[\begin{array}{l}I \\ D\end{array}\right] v$. Transform the latter into a DV-representation that satisfies the assumptions of Step 1 by applying feedback $F=-\left(D+D^{\top}\right)^{-1} C$ and transformation $\left(D+D^{\top}\right)^{-\frac{1}{2}}$ in the driving variable space (see Lemma 5.1). This yields the 'normalized' DV-representation

$$
\begin{aligned}
\dot{x} & =\left(A-B\left(D+D^{\top}\right)^{-1} C\right) x+B\left(D+D^{\top}\right)^{-\frac{1}{2}} v \\
{\left[\begin{array}{l}
u \\
y
\end{array}\right] } & =\left[\begin{array}{c}
-\left(D+D^{\top}\right)^{-1} C \\
C-D\left(D+D^{\top}\right)^{-1} C
\end{array}\right] x+\left[\begin{array}{c}
\left(D+D^{\top}\right)^{-\frac{1}{2}} \\
D\left(D+D^{\top}\right)^{-\frac{1}{2}}
\end{array}\right] v .
\end{aligned}
$$

Now, apply Steps $2-8$ to this representation. The Hamiltonian matrix $H$ in this case is computed to be equal to the Hamiltonian matrix (7) of Sorensen's algorithm. In Step 8 we arrive at a DV-representation of the reduced order behavior $\hat{\mathfrak{B}}: \dot{x}_{1}=$ $\hat{A} x_{1}+\hat{B} v, \quad\left[\begin{array}{l}u \\ y\end{array}\right]=\left[\begin{array}{c}\hat{C}_{1} \\ \hat{C}_{2}\end{array}\right] x_{1}+\left[\begin{array}{c}\left(D+D^{\top}\right)^{-\frac{1}{2}} \\ D\left(D+D^{\top}\right)^{-\frac{1}{2}}\end{array}\right] v$. As a last step, we transform this DV-representation back to an input-state-output representation: note that $v=\left(D+D^{\top}\right)^{\frac{1}{2}} u-\left(D+D^{\top}\right)^{\frac{1}{2}} \hat{C}_{1} x_{1}$. Thus we obtain the following input-state-output 
representation of $\hat{\mathfrak{B}}$ :

$$
\begin{aligned}
\dot{x}_{1} & =\left(\hat{A}-\hat{B}\left(D+D^{\top}\right)^{\frac{1}{2}} \hat{C}_{1}\right) x_{1}+\hat{B}\left(D+D^{\top}\right)^{\frac{1}{2}} u, \\
y & =\left(\hat{C}_{2}-D\left(D+D^{\top}\right)^{\frac{1}{2}} \hat{C}_{1}\right) x_{1}+D u .
\end{aligned}
$$

2. The bounded real case: The DV-representation $\dot{x}=A x+B v,\left[\begin{array}{l}u \\ y\end{array}\right]=\left[\begin{array}{l}0 \\ C\end{array}\right] x+$ $\left[\begin{array}{c}I \\ D\end{array}\right] v$ should again be transformed into a DV-representation satisfying the assumptions of Step 1. For this, we this time apply state feedback $F=\left(I-D^{\top} D\right)^{-1} D^{\top} C$, and transformation $\left(I-D^{\top} D\right)^{-\frac{1}{2}}$ in the driving variable space. The Hamiltonian matrix in this case becomes

$$
H=\left[\begin{array}{ll}
A+B\left(I-D^{\top} D\right)^{-1} D^{\top} C & B\left(I-D^{\top} D\right)^{-1} B^{\top} \\
-C^{\top}\left(I+D\left(I-D^{\top} D\right)^{-1} D^{\top}\right) C & -\left(A+B\left(I-D^{\top} D\right)^{-1} D^{\top} C\right)^{\top}
\end{array}\right] .
$$

As in the strictly passive case, Algorithm 1 leads to a DV-representation of a reduced order behavior $\hat{\mathfrak{B}}$, which can be transformed into an input-state-output representation of the same behavior.

Remark 6.5 Of course, a similar algorithmic procedure can be given for the alternative problem in which the original system $\mathfrak{B}$ is strictly dissipative on $\mathbb{R}^{+}$, and with $\mathfrak{B}^{\prime}$ a subbehavior of the stable part of $\mathfrak{B}^{*}$, and where it is required to find a reduced order behavior $\hat{\mathfrak{B}}$ such that $\hat{\mathfrak{B}}^{*}$ is a subbehavior of the stable part of $\hat{\mathfrak{B}}^{*}$. Again, the details are left to the reader.

Example 6.6 In this example, we illustrate the application of Algorithm 1 to the case of a third order strictly passive system to be reduced to a second-order model. The original system is a single input, single output system with transfer function $g(\xi)=n(\xi) / \mathrm{d}(\xi)$, with $\mathrm{d}(\xi)=9+77 \xi+39 \xi^{2}+7 \xi^{3}$ and $n(\xi)=196+137 \xi+56 \xi^{2}+7 \xi^{3}$. The spectral zeros are the zeros of $g(-\xi)+g(\xi)$, and are $\pm 1, \pm 2$, and \pm 3 . We plan to reduce it by retaining $\mathfrak{B}^{\prime}$, the trajectories of minimal dissipation corresponding to the spectral zeroes 2 and 3. We construct a minimal DV-representation of this system, with $w=(u, y)$, and driving variable $v$. Such DV-representation is given by the matrices

$$
\hat{A}=\left[\begin{array}{ccc}
0 & 1 & 0 \\
0 & 0 & 1 \\
-\frac{9}{7} & -11 & -\frac{39}{7}
\end{array}\right], \hat{B}=\left[\begin{array}{l}
0 \\
0 \\
1
\end{array}\right], \hat{C}=\left[\begin{array}{ccc}
0 & 0 & 0 \\
\frac{187}{7} & \frac{60}{7} & \frac{17}{7}
\end{array}\right], \hat{D}=\left[\begin{array}{l}
1 \\
1
\end{array}\right]
$$

In Step 1 of the algorithm we apply state feedback and a transformation in the driving variable space to obtain a new DV representation given by the matrices

$$
A=\left[\begin{array}{ccc}
0 & 1 & 0 \\
0 & 0 & 1 \\
-\frac{205}{14} & -\frac{107}{7} & -\frac{95}{14}
\end{array}\right], B=\left[\begin{array}{c}
0 \\
0 \\
\frac{1}{\sqrt{2}}
\end{array}\right], C=\left[\begin{array}{ccc}
-\frac{187}{14} & -\frac{30}{7} & -\frac{17}{14} \\
\frac{187}{14} & \frac{30}{7} & \frac{17}{14}
\end{array}\right], D=\left[\begin{array}{c}
\frac{1}{\sqrt{2}} \\
\frac{1}{\sqrt{2}}
\end{array}\right]
$$


We construct the Hamiltonian $H$ corresponding to this representation, and following Step 2 of the algorithm we compute $\left[\begin{array}{c}X_{1} \\ Y_{1}\end{array}\right]$ with

$$
X_{1}:=\left[\begin{array}{ccc}
\frac{7}{2,080} & \frac{7}{1,125} & \frac{7}{528} \\
\frac{21}{2,080} & \frac{14}{1,125} & \frac{7}{528} \\
\frac{63}{2,080} & \frac{28}{1,125} & \frac{7}{528}
\end{array}\right], Y_{1}:=\left[\begin{array}{ccc}
\frac{211}{56} & \frac{107}{21} & \frac{223}{28} \\
\frac{195}{56} & \frac{92}{21} & \frac{145}{28} \\
1 & 1 & 1
\end{array}\right]
$$

whose columns are the eigenvectors of $H$ corresponding to the eigenvalues 3,2 , 1, in this order. Note that the first two columns of $\left[\begin{array}{c}X_{1} \\ Y_{1}\end{array}\right]$ yield the given $\mathfrak{B}^{\prime}$. It is easy to verify that the matrix $X_{1}$ is nonsingular, and that $X_{1}^{\top} Y_{1}$ has the Cholesky factorization $P^{\top} P$, with

$$
P:=\left[\begin{array}{ccc}
\frac{\sqrt{5}}{8} & \frac{11}{15 \sqrt{5}} & \frac{7}{8 \sqrt{5}} \\
0 & \frac{2}{15 \sqrt{5}} & \frac{19}{48 \sqrt{5}} \\
0 & 0 & \frac{\sqrt{7}}{48}
\end{array}\right]
$$

The transformation matrix $S$ computed in Step 4 is

$$
S=X_{1} P^{-1}=\left[\begin{array}{ccc}
\frac{7}{260 \sqrt{5}} & \frac{133}{1,560 \sqrt{5}} & \frac{1,057 \sqrt{7}}{85,800} \\
\frac{21}{260 \sqrt{5}} & \frac{7}{312 \sqrt{5}} & -\frac{1,561 \sqrt{7}}{85,800} \\
\frac{63}{260 \sqrt{5}} & -\frac{623}{1,560 \sqrt{5}} & \frac{1,453 \sqrt{7}}{85,800}
\end{array}\right]
$$

After the transformation of the matrices of the original DV representation (Step 5) and the truncation of the matrices to the first two components of the state (Step 6), we obtain the DV representation induced by

$$
A_{r}:=\left[\begin{array}{rr}
-\frac{17}{5} & -\frac{3}{10} \\
\frac{26}{5} & -\frac{89}{40}
\end{array}\right], B_{r}:=\left[\begin{array}{c}
4 \sqrt{\frac{2}{5}} \\
-\frac{13}{2 \sqrt{10}}
\end{array}\right], C_{r}:=\left[\begin{array}{cc}
-\frac{1}{\sqrt{5}} & -\frac{17}{26 \sqrt{5}}-\frac{\sqrt{5}}{52} \\
\frac{1}{\sqrt{5}} & \frac{17}{26 \sqrt{5}}+\frac{\sqrt{5}}{52}
\end{array}\right], D_{r}:=\left[\begin{array}{l}
\frac{1}{\sqrt{2}} \\
\frac{1}{\sqrt{2}}
\end{array}\right] .
$$

It is easy to verify that the system obtained in this way is controllable, so that the Kalman decomposition of Step 7 is not necessary. $\hat{\mathfrak{B}}=\mathfrak{B}_{\mathrm{DV}}\left(A_{r}, B_{r}, C_{r}, D_{r}\right)_{\text {ext }}$ is strictly passive. It can be verified that the eigenvalues of the reduced order Hamiltonian matrix are $\pm 2, \pm 3$ and that the antistable part of $\hat{\mathfrak{B}}^{*}$ is in fact equal to the given $\mathfrak{B}^{\prime}$.

By eliminating the driving variable and the state from $\mathfrak{B}_{\mathrm{DV}}\left(A_{r}, B_{r}, C_{r}, D_{r}\right)$, we obtain the transfer function from input to output of the reduced order system, given by $\hat{g}(\xi)=\hat{n}(\xi) / \hat{d}(\xi)$, with $\hat{d}(\xi)=9+20 \xi+4 \xi^{2}$ and $\hat{n}(\xi)=64+25 \xi+4 \xi^{2}$. 


\subsection{Rational interpolation at the spectral zeros}

In this subsection, we will show that the transfer matrix associated with any reduced order system $\hat{\mathfrak{B}}$ obtained in Algorithm 1 is in fact a solution of a tangential Nevanlinna rational interpolation problem. Let $\mathfrak{B} \in \mathfrak{L}_{\text {contr }}^{\mathrm{w}}$ be represented by the minimal DV-representation $\mathfrak{B}_{\mathrm{DV}}(A, B, C, D)$, with $D^{\top} \Sigma C=0$ and $D^{\top} \Sigma D=I$, and let $G(\xi):=$ $D+C(\xi I-A)^{-1} B$ be its transfer matrix from driving variable to manifest variable. Let $\mathfrak{B}^{\prime}$ be a given subbehavior of $\left(\mathfrak{B}^{*}\right)_{\text {antistable }}$, and let $\hat{\mathfrak{B}}:=\mathfrak{B}_{\mathrm{DV}}(\hat{A}, \hat{B}, \hat{C}, \hat{D})_{\text {ext }}$ be any reduced order system obtained from Algorithm 1 . Let $\hat{G}(\xi):=\hat{D}+\hat{C}(\xi I-\hat{A})^{-1} \hat{B}$.

As noted before, $\mathfrak{B}^{\prime}$ is associated with a unique $\mathrm{k}$-dimensional $H$-invariant subspace $\mathcal{V}$ of the antistable subspace $X_{+}(H)$ of $H$. In the remainder of this section, for simplicity we assume that the eigenvalues $\lambda_{1}, \lambda_{2}, \ldots, \lambda_{\mathrm{k}}$ of the restriction $\left.H\right|_{\mathcal{V}}$ are distinct. In that case, the matrix $M_{11}$ in Step 2 of Algorithm 1 (being a matrix representation of this restriction) can be diagonalized: there exists a nonsingular complex $\mathrm{k} \times \mathrm{k}$ matrix $U$ such that $M_{11}=U^{-1} \Lambda U$, with $\Lambda:=\operatorname{diag}\left(\lambda_{1}, \lambda_{2}, \ldots, \lambda_{\mathrm{k}}\right)$. Let $P$ be a nonsingular upper triangular matrix from Step 3 of Algorithm 1, say

$$
P=\left[\begin{array}{cc}
P_{11} & P_{12} \\
0 & P_{22}
\end{array}\right] \text {. }
$$

Consider the complex $\mathrm{n} \times \mathrm{k}$ matrix $\left[\begin{array}{c}P_{11} \\ 0\end{array}\right] U^{-1}$ and let $p_{1}, p_{2}, \ldots, p_{\mathrm{k}} \in \mathbb{C}^{\mathrm{n}}$ be its $\mathrm{k}$ columns. Finally, let $(\bar{A}, \bar{B}, \bar{C}, \bar{D})$ be the system matrices obtained after applying the similarity transformation $S$ in Step 5, and let $\bar{H}$ denote the corresponding Hamiltonian matrix. We will now show that the reduced order transfer matrix $\hat{G}(\xi)$ is a solution of a rational tangential interpolation problem at the interpolation points $\lambda_{1}, \lambda_{2}, \ldots, \lambda_{\mathrm{k}}$ and their mirror images in the imaginary axis $-\bar{\lambda}_{1},-\bar{\lambda}_{2}, \ldots,-\bar{\lambda}_{\mathrm{k}}$, with data given by the values $G\left(\lambda_{i}\right), G\left(-\bar{\lambda}_{i}\right)$, and the vectors $p_{i}$ :

Theorem 6.7 1. For $i=1,2, \ldots, \mathrm{k}$, assume $\lambda_{i}$ is not an eigenvalue of $A$ and not an eigenvalue of $\bar{A}_{11}$. Define

$$
v_{i}:=\bar{B}^{\top} p_{i}, \quad w_{i}:=G\left(\lambda_{i}\right) v_{i}
$$

Then $\hat{G}(\xi)$ satisfies $w_{i}=\hat{G}\left(\lambda_{i}\right) v_{i}(i=1,2, \ldots, \mathrm{k})$.

2. For $i=1,2, \ldots, \mathrm{k}$, assume $-\bar{\lambda}_{i}$ is not an eigenvalue of $A$ and not an eigenvalue of $\bar{A}_{11}$. Define

$$
z_{i}:=p_{i}^{*} \bar{C}^{\top} \Sigma, \quad y_{i}:=z_{i} G\left(-\bar{\lambda}_{i}\right)
$$

Then $\hat{G}(\xi)$ satisfies $y_{i}=z_{i} \hat{G}\left(-\bar{\lambda}_{i}\right)(i=1,2, \ldots, \mathrm{k})$.

Note that in the case that the driving variable is one-dimensional, equivalently, the input cardinality of the systems $\mathfrak{B}$ and $\hat{\mathfrak{B}}$ is equal to one, then $\hat{G}\left(\lambda_{i}\right)=G\left(\lambda_{i}\right)$ for $i=1,2, \ldots, \mathrm{k}$, so the transfer matrix $\hat{G}$ of the reduced order system actually interpolates the values $G\left(\lambda_{i}\right)$ at the interpolation points $\lambda_{1}, \lambda_{2}, \ldots, \lambda_{\mathrm{k}}$. 
Proof (1) First note that each $p_{i}$ is of the form $\left(p_{i 1}^{\top}, 0\right)^{\top}$, with $p_{i 1} \in \mathbb{R}^{\mathrm{k}}$ the $i$ th column of $P_{11} U^{-1}$. Also note that $\left(p_{i}^{\top}, p_{i}^{\top}\right)^{\top} \in \mathbb{R}^{2 \mathrm{n}}$ is an eigenvector of $\bar{H}$ with eigenvalue $\lambda_{i}$ $(i=1,2, \ldots, \mathrm{k})$. This implies $\left(\bar{A}+\bar{B} \bar{B}^{\top}\right) p_{i}=\lambda_{i} p_{i}$, so $\left(\lambda_{i} I-\bar{A}\right)^{-1} \bar{B} \bar{B}^{\top} p_{i}=p_{i}$. This immediately implies

$$
G\left(\lambda_{i}\right) \bar{B}^{\top} p_{i}=\left(\bar{D} \bar{B}^{\top}+\bar{C}\right) p_{i}
$$

On the other hand, with $\left(\bar{A}_{11}, \bar{B}_{1}, \bar{C}_{1}, \bar{D}\right)$ the truncated system obtained in Step 6, we have $\left(A_{11}+B_{1} B_{1}^{\top}\right) p_{i 1}=\lambda_{i} p_{i 1}$, so $\left(\lambda_{i} I-A_{11}\right)^{-1} B_{1} B_{1}^{\top} p_{i 1}=p_{i 1}$, which implies that

$$
G_{1}\left(\lambda_{i}\right) B_{1}^{\top} p_{i 1}=\left(\bar{D} B_{1}^{\top}+C_{1}\right) p_{i 1}
$$

where $G_{1}(\xi):=D+C_{1}\left(I \xi-A_{11}\right)^{-1} B_{1}$ is the transfer matrix associated with the truncated system. Combining (28) and (29), upon noting that $\bar{B}^{\top} p_{i}=B_{1}^{\top} p_{i 1}$ and $\bar{C} p_{i}=C_{1} p_{i 1}$ we obtain that $G\left(\lambda_{i}\right) \bar{B}^{\top} p_{i}=G_{1}\left(\lambda_{i}\right) \bar{B}^{\top} p_{i}$. The proof is then completed by noting that $\hat{G}=G_{1}$.

(2) In the same way as above we obtain $\bar{C}^{\top} \Sigma \bar{C} p_{i}=\left(\lambda_{i} I+\bar{A}^{\top}\right) p_{i}$, which implies $\bar{B}^{\top} p_{i}=\bar{B}^{\top}\left(\lambda_{i} I+\bar{A}^{\top}\right)^{-1} \bar{C}^{\top} \Sigma \bar{C} p_{i}$. Since $\bar{D}^{\top} \Sigma \bar{C}=0$, this yields $p_{i}^{*} \bar{B}=$ $-p_{i}^{*} \bar{C}^{\top} \Sigma G\left(-\bar{\lambda}_{i}\right)$. Similarly, we obtain $p_{i 1}^{*} B_{1}=-p_{i 1}^{*} C_{1}^{\top} \Sigma G_{1}\left(-\bar{\lambda}_{i}\right)$. Again note that $\bar{B}^{\top} p_{i}=B_{1}^{\top} p_{i 1}$ and $\bar{C} p_{i}=C_{1} p_{i 1}$. We conclude that $p_{i}^{*} \bar{C}^{\top} \Sigma G\left(-\bar{\lambda}_{i}\right)=p_{i}^{*} \bar{C}^{\top} \Sigma \hat{G}$ $\left(-\bar{\lambda}_{i}\right)$.

The above shows that Algorithm 1 in fact computes, for the given transfer matrix $G(\xi)=D+C(\xi I-A)^{-1} B$, a transfer matrix $\hat{G}(\xi)$ representing a reduced order behavior which is strictly $\Sigma$-dissipative on $\mathbb{R}^{-}$, and which interpolates $G\left(\lambda_{i}\right)$ and $G\left(-\bar{\lambda}_{i}\right)$ in the sense that $\hat{G}\left(\lambda_{i}\right) v_{i}=G\left(\lambda_{i}\right) v_{i}$ with $v_{i}:=\bar{B}^{\top} p_{i}$ and $z_{i} \hat{G}\left(-\bar{\lambda}_{i}\right)=$ $z_{i} G\left(-\bar{\lambda}_{i}\right)$ with $z_{i}:=p_{i}^{*} \bar{C}^{\top} \Sigma(i=1,2, \ldots, \mathrm{k})$.

Thus, Algorithm 1 solves a Nevanlinna type tangential interpolation problem, with interpolation point at $2 \mathrm{k}$ spectral zeros of the original system, $\mathrm{k}$ of which are antistable, and the remaining $\mathrm{k}$ their mirror images in the imaginary axis. We note that the vectors $v_{i}=\bar{B}^{\top} p_{i}$ are in fact zero directions of the rational matrix $G^{\top}(-\xi) \Sigma G(\xi)$, in the sense that $G^{\top}\left(-\lambda_{i}\right) \Sigma G\left(\lambda_{i}\right) v_{i}=0$. Indeed, this is easily verified using $G(\xi)=$ $\bar{C}(\xi I-\bar{A})^{-1} \bar{B}+\bar{D},\left(\bar{A}+\bar{B} \bar{B}^{\top}\right) p_{i}=\lambda_{i} p_{i}$ and $\bar{C}^{\top} \Sigma \bar{C} p_{i}-\bar{A}^{\top} p_{i}=\lambda_{i} p_{i}$. Finally, the $z_{i}$ are related to the $v_{i}$ via $v_{i}=-G^{\top}\left(-\lambda_{i}\right) z_{i}^{*}$.

Remark 6.8 The fact that $\hat{G}$ interpolates the original transfer matrix $G$ at 2 k spectral zeros makes us (formally) consider $\hat{\mathfrak{B}}$ to be an approximation of $\mathfrak{B}$. It can be shown that if both $G$ and $\hat{G}$ are inner, then the difference $\|G-\hat{G}\|_{\mathcal{H}_{\infty}}$ provides an upper bound to the gap between the behaviors $\mathfrak{B} \cap \mathfrak{L}_{2}$ and $\hat{\mathfrak{B}} \cap \mathfrak{L}_{2}$, considered as subspaces of the Hilbert space $\mathfrak{L}_{2}\left(\mathbb{R}, \mathbb{R}^{\mathrm{w}}\right)$. This makes it possible to prove that if one applies the classical method of balanced truncation to an "inner" DV-representation of $\mathfrak{B}$, then the gap between $\mathfrak{B}$ and $\hat{\mathfrak{B}}$ is bounded from above by "twice the sum of the remaining Hankel singular values". Up to now, for the reduction method elaborated in this paper such error bound has not been established. 
Remark 6.9 In this section, we have assumed that the eigenvalues $\lambda_{1}, \lambda_{2}, \ldots, \lambda_{\mathrm{k}}$ of the restriction $\left.H\right|_{\mathcal{V}}$ are distinct, in which case the matrix $M_{11}$ in Step 2 of Algorithm 1 can be diagonalized. In the general case, with coinciding eigenvalues, the reduced order transfer matrix $\hat{G}(\xi)$ can still be shown to solve a tangential interpolation problem. In this case, also the derivatives $\frac{\mathrm{d}}{\mathrm{d} s} \hat{G}(\xi), \frac{\mathrm{d}^{2}}{\mathrm{~d}^{2} s} \hat{G}(\xi)$. . up to some order (depending on the geometric multiplicities of the eigenvalues $\lambda_{i}$ ) are involved in the interpolation at the $\lambda_{i}$ 's in certain directions. We omit the details.

\section{Conclusions}

In this paper, we have introduced and resolved the problem of dissipativity preserving model reduction by retention of trajectories of minimal dissipation. The problem is to find, for a given dissipative behavior $\mathfrak{B}$ of McMillan degree $n$, and a degree $\mathrm{k}$ subbehavior $\mathfrak{B}^{\prime}$ of the subbehavior of minimal dissipation, a dissipative approximative behavior $\hat{\mathfrak{B}}$ of McMillan degree $\mathrm{k}$ whose subbehavior of minimal dissipation is contained in $\mathfrak{B}^{\prime}$. This means that the approximative behavior $\hat{\mathfrak{B}}$ "inherits" trajectories of minimal dissipation from $\mathfrak{B}$. We have given an algorithmic procedure to compute $\hat{\mathfrak{B}}$ from $\mathfrak{B}$ in the case that $\mathfrak{B}$ is given in driving variable representation. The algorithm is based on analysis of invariant subspaces of a Hamiltonian matrix, and on truncation of a state space model obtained after suitable state space transformation. The use of the Hamiltonian matrix for computing an approximative system is reminiscent to the work of Sorensen [19], where a Hamiltonian matrix is used to compute a passive approximation of a given input/state/output system. Indeed, the work in the present paper can be seen as a behavioral formulation and interpretation of the ideas of Antoulas [2] and Sorensen [19] on passivity preserving model reduction using rational interpolation. Of course, the results in our paper are valid for general supply rates. In our paper we show, a fortiori, that the transfer matrices of our reduced order behaviors are solutions of certain tangential Nevanlinna interpolation problems, with interpolation points at the spectral zeros of the original behavior (see also [11]).

The algorithm given in this paper computes, for a given behavior in driving variable representation, a reduced order behavior $\hat{\mathfrak{B}}$, also given in driving variable representation. Of course, in a certain sense this choice of representation is arbitrary, and one would like to have algorithms to compute reduced order behaviors for different types of representations as well, such as kernel representations, image representations, etc. For output nulling representations, a theory analogous to the one presented in this paper for driving variable representations, including an algorithm that computes for a given behavior in output nulling representation a reduced order behavior $\hat{\mathfrak{B}}$ in output nulling representation, is available. For this we refer to [9].

As for kernel representations and image representations, at this moment the only way to compute reduced order behaviors that solve our Main Problem is first to pass from such representation to a driving variable representation or an output nulling representation by applying some realization procedure (see for example $[16,18,26]$ ), next to apply the results in this paper or in [9], and finally to apply an elimination procedure to arrive at a kernel or image representation of the reduced order behavior (see [16]). Improvements of this are the subject of current research. 
Acknowledgments The authors would like to thank Prof. A. C. Antoulas for some extremely useful discussions on the topic of this paper.

Open Access This article is distributed under the terms of the Creative Commons Attribution Noncommercial License which permits any noncommercial use, distribution, and reproduction in any medium, provided the original author(s) and source are credited.

\section{Appendix: Basics of driving-variable and output nulling representations}

As already noted in Sect. 2, linear differential systems often result as external behavior of systems with latent variables. Two particular instances of such latent variable representations are systems with driving variables, and output nulling systems. In these latent variable systems, the latent variable in fact satisfies the axiom of state. In this Appendix, we have collected the basic material on driving variable and output nulling representations.

\subsection{Driving-variable representations}

Let $A \in \mathbb{R}^{\mathrm{n} \times \mathrm{n}}, B \in \mathbb{R}^{\mathrm{n} \times \mathrm{v}}, C \in \mathbb{R}^{\mathrm{w} \times \mathrm{n}}, D \in \mathbb{R}^{\mathrm{w} \times \mathrm{v}}$, and consider the equations

$$
\begin{aligned}
\dot{x} & =A x+B v \\
w & =C x+D v .
\end{aligned}
$$

These equations represent the full behavior

$$
\begin{aligned}
& \mathfrak{B}_{D V}(A, B, C, D) \\
& \quad:=\left\{(w, x, v) \in \mathfrak{C}^{\infty}\left(\mathbb{R}, \mathbb{R}^{\mathrm{w}}\right) \times \mathfrak{C}^{\infty}\left(\mathbb{R}, \mathbb{R}^{\mathrm{n}}\right) \times \mathfrak{C}^{\infty}\left(\mathbb{R}, \mathbb{R}^{\mathrm{v}}\right) \mid(30) \text { hold }\right\} .
\end{aligned}
$$

In we interpret $w$ as manifest variable and $(x, v)$ as latent variable, then $\mathfrak{B}_{\mathrm{DV}}(A, B$, $C, D)$ is a latent variable representation of its external behavior

$$
\begin{array}{r}
\mathfrak{B}_{D V}(A, B, C, D)_{\mathrm{ext}}=\left\{w \in \mathfrak{C}^{\infty}\left(\mathbb{R}, \mathbb{R}^{\mathrm{w}}\right) \mid \exists x \in \mathfrak{C}^{\infty}\left(\mathbb{R}, \mathbb{R}^{\mathrm{n}}\right) \text { and } v \in \mathfrak{C}^{\infty}\left(\mathbb{R}, \mathbb{R}^{\mathrm{v}}\right)\right. \\
\text { such that } \left.(w, x, v) \in \mathfrak{B}_{D V}(A, B, C, D)\right\} .
\end{array}
$$

The variable $x$ is in fact a state variable, the variable $v$ is free, and is called the driving variable.

If $\mathfrak{B}=\mathfrak{B}_{\mathrm{DV}}(A, B, C, D)_{\text {ext }}$ then we call $\mathfrak{B}_{\mathrm{DV}}(A, B, C, D)$ a driving variable representation of $\mathfrak{B}$. A driving variable representation $\mathfrak{B}_{\mathrm{DV}}(A, B, C, D)$ of $\mathfrak{B}$ is called minimal if the state dimension $\mathrm{n}$ and the driving variable dimension $\mathrm{v}$ are minimal over all such driving variable representations. In the following, let $n(\mathfrak{B})$ and $m(\mathfrak{B})$ denote the McMillan degree of $\mathfrak{B}$, and the input cardinality of $\mathfrak{B}$, respectively. The following result is well known:

Proposition 8.1 Let $\mathfrak{B} \in \mathfrak{L}^{\mathrm{w}}$ be given. Denote $\mathrm{n}=\mathrm{n}(\mathfrak{B})$ and $\mathrm{m}=\mathrm{m}(\mathfrak{B})$. Then

1. There exists matrices $A \in \mathbb{R}^{\mathrm{n} \times \mathrm{n}}, B \in \mathbb{R}^{\mathrm{n} \times \mathrm{m}}, C \in \mathbb{R}^{\mathrm{w} \times \mathrm{n}}, D \in \mathbb{R}^{\mathrm{w} \times \mathrm{m}}$ such that $\mathfrak{B}_{\mathrm{DV}}(A, B, C, D)$ is a minimal driving variable representation of $\mathfrak{B}$, 
2. If $\mathfrak{B}_{\mathrm{DV}}(A, B, C, D)$ represents $\mathfrak{B}$, then it is a minimal representation if and only if $D$ is injective and the pair $(C+D F, A+B F)$ is observable for all $F$,

3. If $\mathfrak{B}_{\mathrm{DV}}(A, B, C, D)$ is a minimal representation of $\mathfrak{B}$, then $\mathfrak{B}_{\mathrm{DV}}\left(A^{\prime}, B^{\prime}, C^{\prime}, D^{\prime}\right)$ is a minimal representation of $\mathfrak{B}$ if and only if there exist invertible matrices $S$ and $R$ and a matrix $F$ such that

$$
\left(A^{\prime}, B^{\prime}, C^{\prime}, D^{\prime}\right)=\left(S^{-1}(A+B F) S, S^{-1} B R,(C+D F) S, D R\right) .
$$

Proof A proof of (1) is given in Section 3 of [18]. For a proof of (2), see [18], Corollary 4.2. For (3) we refer to [26], Theorem 7.2 (see also Remark 8.3 in that paper).

The next proposition states that in order to compute a minimal driving variable representation from a given one, we can use state feedback.

Proposition 8.2 Let $\mathfrak{B} \in \mathfrak{L}^{\mathrm{w}}$ and let $\mathfrak{B}_{\mathrm{DV}}(A, B, C, D)$ be a driving variable representation of $\mathfrak{B}$, with $D$ injective. Define $F:=-\left(D^{\top} D\right)^{-1} D^{\top} C$. Then there is a nonsingular matrix $S$ such that $S^{-1}(A+B F) S=\left[\begin{array}{cc}A_{11}^{\prime} & 0 \\ A_{21}^{\prime} & A_{22}^{\prime}\end{array}\right], S^{-1} B=\left[\begin{array}{l}B_{1}^{\prime} \\ B_{2}^{\prime}\end{array}\right]$, $(C+D F) S=\left[\begin{array}{ll}C_{1}^{\prime} & 0\end{array}\right]$ such that

1. The pair $\left(C_{1}^{\prime}+D F^{\prime}, A_{11}^{\prime}+B_{1}^{\prime} F^{\prime}\right)$ is observable for all $F^{\prime}$,

2. $B_{\mathrm{DV}}\left(A_{11}^{\prime}, B_{1}^{\prime}, C_{1}^{\prime}, D\right)_{\mathrm{ext}}=\mathfrak{B}_{\mathrm{DV}}(A, B, C, D)_{\mathrm{ext}}$.

Consequently, $\mathfrak{B}_{\mathrm{DV}}\left(A_{11}^{\prime}, B_{1}^{\prime}, C_{1}^{\prime}, D\right)$ is a minimal driving variable representation of $\mathfrak{B}$.

Proof Let $\mathcal{V}^{*}$ be the weakly unobservable subspace of $(A, B, C, D)$ (see [22], Section 7.3). By [22], Exercise 7.5, $\mathcal{V}^{*}$ is equal to the unobservable subspace of the pair $(C+D F, A+B F)$, with $F=-\left(D^{\top} D\right)^{-1} D^{\top} C$. With respect to a basis adapted to $\mathcal{V}^{*}, A+B F, C+D F$ and $B$ have matrices partitioned as claimed above. By construction, the weakly unobservable subspace of $\left(A_{11}^{\prime}, B_{1}^{\prime}, C_{1}^{\prime}, D\right)$ is zero and therefore, by [22] Theorem 7.16, statement (1) of the proposition holds.

In order to prove that $B_{\mathrm{DV}}\left(A_{11}^{\prime}, B_{1}^{\prime}, C_{1}^{\prime}, D\right)_{\text {ext }}=\mathfrak{B}_{\mathrm{DV}}(A, B, C, D)_{\text {ext }}$, observe that since coordinate transformations and state feedback do not change the external behavior, we have $\mathfrak{B}_{\mathrm{DV}}\left(S^{-1}(A+B F) S, S^{-1} B,(C+D F) S, D\right)_{\mathrm{ext}}=\mathfrak{B}_{\mathrm{DV}}(A, B, C, D)_{\mathrm{ext}}$. We now prove that $\mathfrak{B}_{\mathrm{DV}}\left(S^{-1}(A+B F) S, S^{-1} B,(C+D F) S, D\right)_{\text {ext }}=B_{\mathrm{DV}}\left(A_{11}^{\prime}, B_{1}^{\prime}\right.$, $\left.C_{1}^{\prime}, D\right)_{\text {ext }}$. The inclusion $\subseteq$ follows immediately. In order to prove the converse inclusion, let $w \in \mathfrak{B}_{\mathrm{DV}}\left(A_{11}^{\prime}, B_{1}^{\prime}, C_{1}^{\prime}, D\right)_{\text {ext }}$. Then there exist $x_{1}, v$ such that

$$
\begin{aligned}
\dot{x}_{1} & =A_{11}^{\prime} x_{1}+B_{1}^{\prime} v \\
w & =C_{1}^{\prime} x_{1}+D v .
\end{aligned}
$$

Then, let $x_{2}$ be any solution of $\dot{x}_{2}=A_{21}^{\prime} x_{1}+A_{22}^{\prime} x_{2}+B_{2}^{\prime} v$. This proves that $w \in$ $\mathfrak{B}_{\mathrm{DV}}\left(S^{-1}(A+B F) S, S^{-1} B,(C+D F) S, D\right)_{\text {ext }}$, so statement (2) of the proposition holds. Finally, the minimality of $\left(A_{11}^{\prime}, B_{1}^{\prime}, C_{1}^{\prime}, D\right)$ as a representation of $\mathfrak{B}$ follows from the fact that $D$ is injective and from statement (1). 
In this paper, in the context of dissipative systems, we mostly work with controllable behaviors, and with the controllable part of a behavior. We now examine under what conditions a behavior represented in driving variable form is controllable.

Proposition 8.3 Let $\mathfrak{B} \in \mathfrak{L}^{\mathrm{w}}$ be given. Then the following statements are equivalent

1. $\mathfrak{B}$ is controllable,

2. There exist matrices $A, B, C$ and $D$ such that $\mathfrak{B}=\mathfrak{B}_{\mathrm{DV}}(A, B, C, D)_{\mathrm{ext}}$ with $(A, B)$ controllable,

3. For every minimal representations $\mathfrak{B}=B_{\mathrm{DV}}(A, B, C, D)_{\mathrm{ext}}$, the pair $(A, B)$ is controllable.

Proof See Theorem 3.11 [23].

Now let $\mathfrak{B}$ be possibly non-controllable, and let $\mathfrak{B}_{\mathrm{DV}}(A, B, C, D)$ be a driving variable representation. The following result shows how to compute a driving variable representation of the controllable part of $\mathfrak{B}$.

Proposition 8.4 Let $\mathfrak{B} \in \mathfrak{L}^{\mathrm{w}}$ and let $\mathfrak{B}_{\mathrm{DV}}(A, B, C, D)$ be a driving variable representation of $\mathfrak{B}$. Then there exists a nonsingular matrix $S$ such that

1. $S^{-1} A S=\left[\begin{array}{cc}\bar{A}_{11} & \bar{A}_{12} \\ 0 & \bar{A}_{22}\end{array}\right], S^{-1} B=\left[\begin{array}{c}\bar{B}_{1} \\ 0\end{array}\right], C S=\left[\begin{array}{ll}\bar{C}_{1} & \bar{C}_{2}\end{array}\right]$,

2. $\left(\bar{A}_{11}, \bar{B}_{1}\right)$ is controllable.

Then, $\mathfrak{B}_{\mathrm{DV}}\left(\bar{A}_{11}, \bar{B}_{1}, \bar{C}_{1}, D\right)$ is a driving variable representation of the controllable part $\mathfrak{B}_{\text {cont }}$ of $\mathfrak{B}$.

Proof First, clearly the full behavior $\mathfrak{B}_{\mathrm{DV}}\left(\bar{A}_{11}, \bar{B}_{1}, \bar{C}_{1}, D\right)$ is controllable. Define $\mathfrak{B}_{0}:=\left\{\left(w,\left(x_{1}, 0\right), v\right) \mid\left(w, x_{1}, v\right) \in \mathfrak{B}_{\mathrm{DV}}\left(\bar{A}_{11}, \bar{B}_{1}, \bar{C}_{1}, D\right)\right\}$. Then $\mathfrak{B}_{0}$ is controllable. Also we have $\mathfrak{B}_{0} \subseteq \mathfrak{B}_{\mathrm{DV}}\left(S^{-1} A S, S^{-1} B, C S, D\right)$, and the input cardinalities of these two behaviors coincide. By [3], Lemma 2.10.3, their controllable parts then coincide, so we have $\mathfrak{B}_{0}=\mathfrak{B}_{\mathrm{DV}}\left(S^{-1} A S, S^{-1} B, C S, D\right)_{\text {cont }}$. Finally, the two operations of taking the controllable part and taking external behavior commute (see [3], Lemma 2.10.4). Thus we obtain $\mathfrak{B}_{\mathrm{DV}}\left(\bar{A}_{11}, \bar{B}_{1}, \bar{C}_{1}, D\right)_{\mathrm{ext}}=\left(\mathfrak{B}_{0}\right)_{\mathrm{ext}}=\left(\mathfrak{B}_{\mathrm{DV}}\right.$ $\left.\left(S^{-1} A S, S^{-1} B, C S, D\right)_{\mathrm{cont}}\right)_{\mathrm{ext}}=\left(\mathfrak{B}_{\mathrm{DV}}\left(S^{-1} A S, S^{-1} B, C S, D\right)_{\mathrm{ext}}\right)_{\mathrm{cont}}=\mathfrak{B}_{\mathrm{cont}}$.

\subsection{Output nulling representations}

Output nulling representations are defined as follows. Let $A \in \mathbb{R}^{\mathrm{n} \times \mathrm{n}}, B \in \mathbb{R}^{\mathrm{n} \times \mathrm{w}}$, $C \in \mathbb{R}^{\mathrm{p} \times \mathrm{n}}, D \in \mathbb{R}^{\mathrm{p} \times \mathrm{w}}$, and consider the equations

$$
\begin{aligned}
& \dot{x}=A x+B w \\
& 0=C x+D w
\end{aligned}
$$

These equations represent the full behavior

$$
\mathfrak{B}_{\mathrm{ON}}(A, B, C, D):=\left\{(w, x) \in \mathfrak{C}^{\infty}\left(\mathbb{R}, \mathbb{R}^{\mathrm{w}}\right) \times \mathfrak{C}^{\infty}\left(\mathbb{R}, \mathbb{R}^{\mathrm{n}}\right) \mid(31) \text { hold }\right\} .
$$

Again, if we interpret $w$ as manifest variable and $x$ as latent variable, then $\mathfrak{B}_{\mathrm{ON}}(A, B$, $C, D)$ is a latent variable representation of its external behavior 
$\mathfrak{B}_{\mathrm{ON}}(A, B, C, D)_{\mathrm{ext}}=\left\{w \in \mathfrak{C}^{\infty}\left(\mathbb{R}, \mathbb{R}^{\mathrm{W}}\right) \mid \exists x \in \mathfrak{C}^{\infty}\left(\mathbb{R}, \mathbb{R}^{\mathrm{n}}\right)\right.$ such that

$$
\left.(w, x) \in \mathfrak{B}_{\mathrm{ON}}(A, B, C, D)\right\} \text {. }
$$

Also here, the variable $x$ is a state variable. If $\mathfrak{B}=\mathfrak{B}_{\mathrm{ON}}(A, B, C, D)_{\text {ext }}$ then we call $\mathfrak{B}_{\mathrm{ON}}(A, B, C, D)$ an output-nulling representation of $\mathfrak{B} \cdot \mathfrak{B}_{\mathrm{ON}}(A, B, C, D)$ is called a minimal output-nulling representation if $\mathrm{n}$ and $\mathrm{p}$ are minimal over all output nulling representations of $\mathfrak{B}$. In the following, let $n(\mathfrak{B})$ and $p(\mathfrak{B})$ denote the McMillan degree of $\mathfrak{B}$, and the output cardinality of $\mathfrak{B}$, respectively. Again, the following is well known:

Proposition 8.5 Let $\mathfrak{B} \in \mathfrak{L}^{\mathrm{w}}$ be given. Denote $\mathrm{n}=\mathrm{n}(\mathfrak{B})$ and $\mathrm{p}=\mathrm{p}(\mathfrak{B})$. Then,

1. There exist matrices $A \in \mathbb{R}^{\mathrm{n} \times \mathrm{n}}, B \in \mathbb{R}^{\mathrm{n} \times \mathrm{w}}, C \in \mathbb{R}^{\mathrm{p} \times \mathrm{n}}, D \in \mathbb{R}^{\mathrm{p} \times \mathrm{w}}$ such that $\mathfrak{B}_{\mathrm{ON}}(A, B, C, D)$ is a minimal output nulling representation of $\mathfrak{B}$,

2. If $\mathfrak{B}_{\mathrm{ON}}(A, B, C, D)$ represents $\mathfrak{B}$, then it is a minimal representation if and only if $D$ is surjective and $(C, A)$ is observable,

3. If $\mathfrak{B}_{\mathrm{ON}}(A, B, C, D)$ is a minimal representation of $\mathfrak{B}$, then $\mathfrak{B}_{\mathrm{ON}}\left(A^{\prime}, B^{\prime}, C^{\prime}, D^{\prime}\right)$ is a minimal representation of $\mathfrak{B}$ if and only if there exist invertible matrices $S$ and $R$ and a matrix $J$ such that

$$
\left(A^{\prime}, B^{\prime}, C^{\prime}, D^{\prime}\right)=\left(S^{-1}(A+J C) S, S^{-1}(B+J D), R C S, R D\right) .
$$

Proof See Theorem 3.20 in [23].

To conclude this Appendix, we recall how driving variable and output nulling representations of a behavior can be used in order to obtain representations for the orthogonal behavior.

Proposition 8.6 Let $\mathfrak{B} \in \mathfrak{L}_{\text {contr }}^{\mathrm{w}}$ and let $\Sigma=\Sigma^{\top} \in \mathbb{R}^{\mathrm{w} \times \mathrm{w}}$ be nonsingular. Then

1. If $\mathfrak{B}_{\mathrm{DV}}(A, B, C, D)$ is a minimal driving variable representation of $\mathfrak{B}$, then $\mathfrak{B}_{\mathrm{ON}}\left(-A^{\top}, C^{\top} \Sigma, B^{\top},-D^{\top} \Sigma\right)$ is a minimal output-nulling representation of $\mathfrak{B}^{\perp_{\Sigma}}$.

2. If $\mathfrak{B}_{\mathrm{ON}}(A, B, C, D)$ is a minimal output-nulling representation of $\mathfrak{B}$, then $\left.\mathfrak{B}_{\mathrm{DV}}\left(-A^{\top}, C^{\top}, \Sigma^{-1} B^{\top},-\Sigma^{-1} D^{\top}\right)\right)$ is a minimal driving variable representation of $\mathfrak{B}^{\perp}$.

Proof See Section VI.A of [31].

\section{References}

1. Antoulas AC (2004) Approximation of large-scale dynamical systems. SIAM Press, Philadelphia

2. Antoulas AC (2005) A new result on passivity-preserving model reduction. Syst Control Lett 54:361374

3. Belur MN (2003) Control in a behavioral context. Dissertation, University of Groningen

4. Bultheel A, van Baren M (1986) Padé techniques for model reduction in linear system theory: a survey. J Comp Appl Math 14:401-438

5. Chen X, Wen JT (1995) Positive realness preserving model reduction with $H_{\infty}$ norm error bounds. IEEE Trans Circuits Syst 42(1):23-29 
6. Kim S-Y, Gopal N, Pillage LT (1994) Time-domain macromodels for VLSI interconnected analysis. IEEE Trans Comput Aided Des Integr Circuit Syst 13:1257-1270

7. Knockaert L, de Zutter D (1999) Passive reduced-order multiport modelling: the Padé-Laguerre, Krylov-Arnoldi-SVD connection. Int J Electron Commun 53:254-260

8. Glover K (1984) All optimal Hankel-norm approximations of linear multivariable systems and their $L_{\infty}$-error bounds. Int J Control 39:1115-1193

9. Minh HB (2009) Model reduction in a behavioral framework. Ph.D. Thesis, University of Groningen, The Netherlands

10. Krajewski W, Lepschy A, Viaro U (1994) Approximation of continuous-time linear systems using Markov parameters and energy indices. IEEE Trans Autom Control 39:2126-2129

11. Fanizza G, Karlsson J, Lindquist A, Nagamune R (2007) Passivity preserving model reduction by analytic interpolation. Linear Algebra Appl 425:608-633

12. Meinsma G (1993) Frequency domain methods in $H_{\infty}$ control. Ph.D. Thesis, University of Twente

13. Moore BC (1981) Principal components analysis in linear systems: controllability, observability, and model reduction. IEEE Trans Autom Control AC-26:17-32

14. Ober R (1991) Balanced parametrization of classes of linear systems. SIAM J Control Optim 29:12511287

15. Opdenacker PC, Jonckheere EA (1988) A contraction mapping preserving balanced reduction scheme and its infinity norm error bounds. IEEE Trans Circuits Syst 35(2):184-189

16. Polderman JW, Willems JC (1997) Introduction to mathematical system theory: a behavioral approach. Springer, Berlin

17. Rapisarda P, Willems JC (1997) State maps for linear systems. SIAM J Control Optim 35(3):10531091

18. Schumacher JM (1988) Linear systems under external equivalence. Linear Algebra Appl 102:1-33

19. Sorensen DC (2005) Passivity preserving model reduction via interpolation of spectral zeros. Syst Control Lett 54:347-360

20. Trentelman HL, Willems JC (1991) The dissipation inequality and the algebraic Riccati equation. In: Bittanti S, Laub AJ, Willems JC (eds) The Riccati equation. Springer, Berlin

21. Trentelman HL, Willems JC (1997) Every storage function is a state function. Syst Control Lett 32:249259

22. Trentelman HL, Stoorvogel AA, Hautus MLJ (2001) Control theory for linear systems. Springer, London

23. Weiland S (1991) Theory of approximation and disturbance attenuation for linear system. Dissertation, University of Groningen

24. Yousuff A, Wagie DA, Skelton RE (1985) Linear system approximation via covariance equivalent realizations. J Math Anal Appl 106:91-115

25. Wang JM, Chu C-C, Yu Q, Kuh ES (2002) On projection-based algorithms for model-order reduction of interconnects. IEEE Trans Circuits Syst I Fundam Theory Appl CAS-49:1564-1585

26. Willems JC (1983) Input-output and state-space representations of finite-dimensional linear timeinvariant systems. Linear Algebra Appl 50:581-608

27. Willems JC, Trentelman HL (1991) The dissipation inequality and the algebraic Riccati equation. The Riccati equation. Springer, Berlin

28. Willems JC (1972) Dissipative dynamical systems-Part I: General theory. Arch Ration Mech Anal 45:321-351

29. Willems JC (1972) Dissipative dynamical systems-Part II: Linear systems with quadratic supply rates. Arch Ration Mech Anal 45:352-393

30. Willems JC, Trentelman HL (1998) On quadratic differential forms. SIAM J Control Optim 36(5):1703-1749

31. Willems JC, Trentelman HL (2002) Synthesis of dissipative systems using quadratic differential forms-Part I. IEEE Trans Autom Control 47(1):53-69

32. Zhou K (1996) Robust and optimal control. Prentice-Hall, New Jersey 